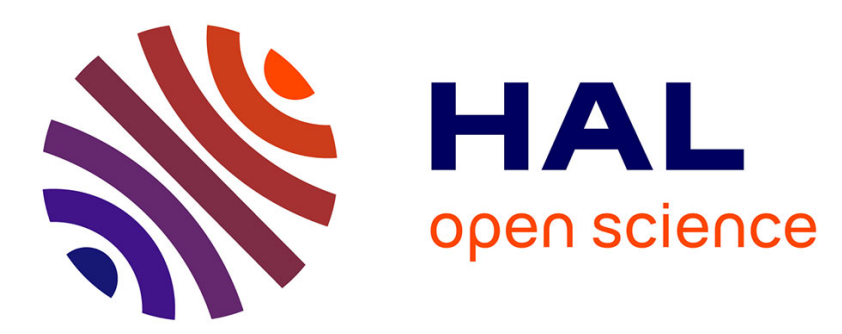

\title{
A limit theorem for the survival probability of a simple random walk among power-law renewal obstacles
}

Julien Poisat, François Simenhaus

\section{To cite this version:}

Julien Poisat, François Simenhaus. A limit theorem for the survival probability of a simple random walk among power-law renewal obstacles. Annals of Applied Probability, 2020, 30 (5). hal$01878052 \mathrm{v} 4$

\section{HAL Id: hal-01878052 \\ https://hal.science/hal-01878052v4}

Submitted on 4 Dec 2019

HAL is a multi-disciplinary open access archive for the deposit and dissemination of scientific research documents, whether they are published or not. The documents may come from teaching and research institutions in France or abroad, or from public or private research centers.
L'archive ouverte pluridisciplinaire HAL, est destinée au dépôt et à la diffusion de documents scientifiques de niveau recherche, publiés ou non, émanant des établissements d'enseignement et de recherche français ou étrangers, des laboratoires publics ou privés. 


\title{
A LIMIT THEOREM FOR THE SURVIVAL PROBABILITY OF A SIMPLE RANDOM WALK AMONG POWER-LAW RENEWAL OBSTACLES
}

\author{
JULIEN POISAT AND FRANÇOIS SIMENHAUS
}

\begin{abstract}
We consider a one-dimensional simple random walk surviving among a field of static soft obstacles: each time it meets an obstacle the walk is killed with probability $1-e^{-\beta}$, where $\beta$ is a positive and fixed parameter. The positions of the obstacles are sampled independently from the walk and according to a renewal process. The increments between consecutive obstacles, or gaps, are assumed to have a power-law decaying tail with exponent $\gamma>0$. We prove convergence in law for the properly rescaled logarithm of the quenched survival probability as time goes to infinity. The normalization exponent is $\gamma /(\gamma+2)$, while the limiting law writes as a variational formula with both universal and non-universal features. The latter involves (i) a Poisson point process that emerges as the universal scaling limit of the properly rescaled gaps and (ii) a function of the parameter $\beta$ that we call asymptotic cost of crossing per obstacle and that may, in principle, depend on the details of the gap distribution. Our proof suggests a confinement strategy of the walk in a single large gap. This model may also be seen as a $(1+1)$-directed polymer among many repulsive interfaces, in which case $\beta$ corresponds to the strength of repulsion, the survival probability to the partition function and its logarithm to the finite-volume free energy.
\end{abstract}

\section{INTRODUCTION AND MODEL}

We consider a one-dimensional simple random walk surviving among a random field of static soft obstacles: each time it meets an obstacle the walk is killed with probability $1-e^{-\beta}$ or survives with probability $e^{-\beta}$, where $\beta$ is a positive and fixed parameter, see Section 1.1 for a precise definition. The increments between consecutive obstacles, or gaps, are assumed to be mutually independent and independent from the walk, with a power-law decaying tail. This is what we refer to as power-law renewal obstacles in the title, see Section 1.3 for a precise definition. To be more precise, we deal with the quenched version of the model, meaning that the positions of the obstacles are frozen and the survival probability is computed only with respect to the law of the random walk. Our main result, Theorem 2.2, states a convergence in law for the properly rescaled logarithm of the quenched survival probability, seen as a random variable with respect to the field of obstacles, as time goes to infinity. The limiting law writes as a variational formula involving (i) a Poisson point process that emerges as the universal scaling limit of the properly rescaled gaps and (ii) a function of the parameter $\beta$ that we call asymptotic cost of crossing per obstacle and that may, in principle, depend on the details of the gap distribution, see the definition of $\lambda(\beta)$ in Proposition 2.1. Even if we offer no path statement for the walk conditioned on

Key words and phrases. Random walks in random obstacles, polymers in random environments, parabolic Anderson model, survival probability, FKG inequalities, Ray-Knight theorems.

Acknowledgements. We thank C. Labbé for suggesting to consider the functional version of our theorem. JP and FS are supported by the ANR/FNS-16-CE93-0003 grant MALIN. JP is also supported by the ANR-17-CE40-0032 grant SWiWS. FS is also supported by the ANR-15-CE40-0020-01 grant LSD.. 
surviving, our proof strongly suggests a confinement strategy according to which the walk remains in a large gap with an appropriate scale. Path localization could be considered as future work.

As we will see in Section 1.2, our model may also be seen as a $(1+1)$-directed polymer among many repulsive interfaces, in which case $\beta$ corresponds to the strength of repulsion. We will take advantage of this connection by using and revisiting some estimates obtained by Caravenna and Pétrélis $[4,5]$ in the case of periodic obstacles. We point out that the logarithm of the survival probability is the finite-volume free energy of the corresponding polymer model.

Outline. Section 1 contains the mathematical definition of the model and a discussion on the relation with other models, such as the directed polymer among multiple interfaces. In Section 2 we state our main result, Theorem 2.2, as well as some auxiliary results. We conclude this section with a list of comments and related open questions. Sections 3 to 6 constitute the proof of the theorem. Key tools are gathered in Section 3. The rest of the proof is split into a lower bound part (Section 4), an upper bound part (Section 5) and a conclusion (Section 6). The more technical proofs are deferred to an appendix.

Notation. In this paper we denote by $\mathbb{N}$ the set of positive integers and $\mathbb{N}_{0}=\mathbb{N} \cup\{0\}$. The letter $C$ is used for the constants whose values are irrelevant and may change from line to line.

1.1. A random walk on $\mathbb{Z}$ among soft obstacles. We consider $S=\left(S_{n}\right)_{n \in \mathbb{N}_{0}}$ a simple random walk on $\mathbb{Z}$ in presence of obstacles. We recall that the increments $\left(S_{n}-S_{n-1}\right)_{n \in \mathbb{N}}$ are independent and identically distributed (i.i.d.) random variables which are uniformly distributed on $\{-1,1\}$, and we shall write $\mathrm{P}_{x}$ for the law of the walk started at $S_{0}=x$, for $x \in \mathbb{Z}$, with the notational simplification $\mathrm{P}_{0}=\mathrm{P}$. The positions of the obstacles are integers and they will be denoted by $\tau=\left\{\tau_{n}\right\}_{n \in \mathbb{N}_{0}}$ with $\tau_{0}=0$. The increments $\left(\tau_{n+1}-\tau_{n}\right)_{n \geq 0}$ may be referred to as gaps.

Let $\beta>0$ be a parameter of the model. Informally, each time the walk meets an obstacle, it is killed with probability $1-e^{-\beta}$ or survives with probability $e^{-\beta}$ independently from the past. The obstacles are called soft, by opposition to hard obstacles, because the walk has a positive chance to survive when sitting on an obstacle. For a precise mathematical definition let us first introduce $\left(\theta_{n}\right)_{n \in \mathbb{N}}$, the clock process recording the times when the random walk visits $\tau$, that is

$$
\theta_{0}=0, \quad \theta_{n+1}=\inf \left\{k>\theta_{n}: S_{k} \in \tau\right\}, \quad n \in \mathbb{N}_{0} .
$$

We enlarge the probability space so as to include a $\mathbb{N}$-valued geometric random variable $\mathcal{N}$ with success parameter $1-e^{-\beta}$. This plays the role of the clock that kills the walk after its $\mathcal{N}$-th meeting with the set of obstacles. We now define $\sigma$ the death time of the walk by

$$
\sigma=\theta_{\mathcal{N}}
$$

Note that our probability law now depends on the parameter $\beta$. We shall write $\mathrm{P}_{x}^{\beta}$ when we want to stress this dependence or omit the superscript when no confusion is possible. Again we may write $\mathrm{P}^{\beta}$ instead of $\mathrm{P}_{0}^{\beta}$. We also point out that $\sigma$ depends on $\tau$ through $\theta$ even if it is not explicit in the notations. 
The hitting times of the walk are defined by

$$
H_{x}=\inf \left\{n \geq 1: S_{n}=x\right\}, \quad x \in \mathbb{Z},
$$

and

$$
H_{\mathbb{Z}^{-}}=\inf \left\{n \geq 1: S_{n} \leq 0\right\} .
$$

In this paper we study the limiting behaviour of the probability that the walk survives up to time $n \in \mathbb{N}$, as $n$ gets large. For convenience, we consider walks that do not visit $\mathbb{Z}^{-}=-\mathbb{N}_{0}$. This extra condition allows to consider obstacles that are indexed by $\mathbb{N}_{0}$ instead of $\mathbb{Z}$ and does not hide anything deep nor change the main idea of the paper. Thus, our survival probability writes

$$
Z_{n}=\mathrm{P}^{\beta}\left(\sigma \wedge H_{\mathbb{Z}^{-}}>n\right), \quad n \in \mathbb{N} .
$$

We stress again that $Z_{n}$ is a function of the environment of obstacles $\left(\tau_{n}\right)_{n \geq 0}$.

1.2. (De)pinning of a $(1+1)$-directed polymer by multiple interfaces. By integrating out $\mathcal{N}$ in (1.5), we obtain

$$
Z_{n}=\mathrm{E}\left[\exp \left(-\beta \sum_{k=1}^{n} 1_{\left\{S_{k} \in \tau\right\}}\right) 1_{\left\{H_{\mathbb{Z}^{-}}>n\right\}}\right] .
$$

This expression links the survival probability to a certain polymer model from statistical mechanics. More precisely, the expression above is the partition function of a $(1+1)$ directed polymer above an impenetrable wall and among many repulsive interfaces. Here, $\left(k, S_{k}\right)_{0 \leq k \leq n}$ plays the role of a polymer with $n$ monomers and the parameter $n$, which is initially a time parameter, becomes the size of the polymer, see Figure 1 . Whenever the polymer touches one of the interfaces, located at the levels $\tau=\left\{\tau_{n}\right\}_{n \in \mathbb{N}_{0}}$, it is penalized by a factor $e^{-\beta}$. Finally, the event $\left\{H_{\mathbb{Z}^{-}}>n\right\}$ reflects the presence of a hard wall at level 0 .

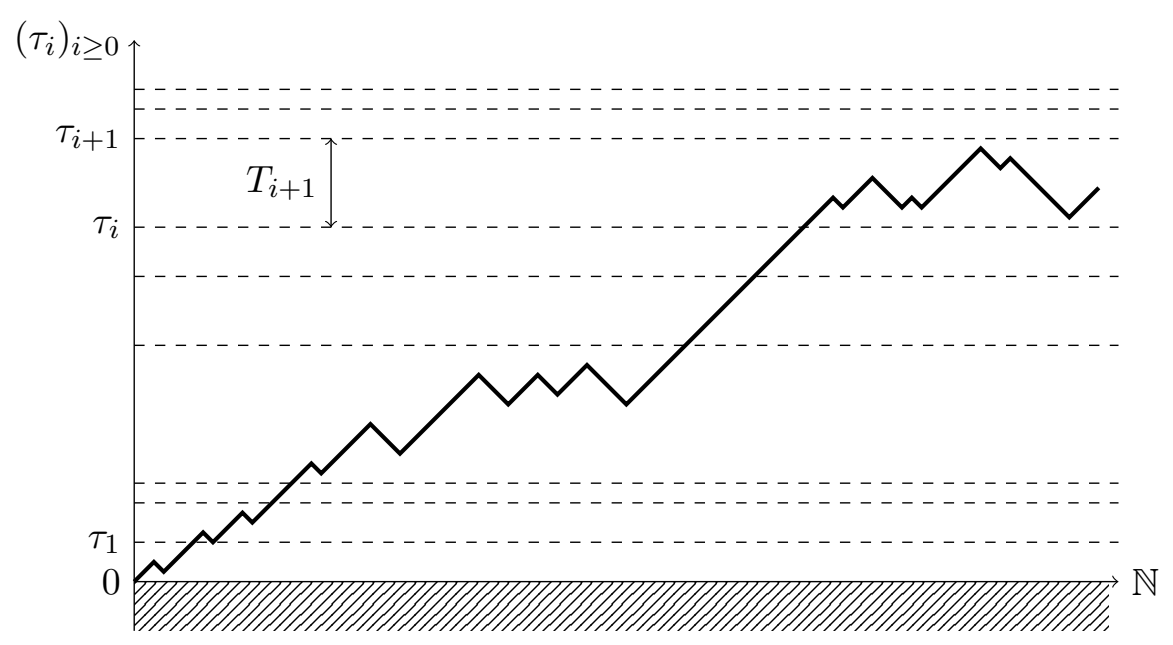

FiguRE 1. Example of a polymer among repulsive interfaces. The dashed lines correspond to the interfaces, the thick one to the polymer and the shaded area to the hard wall.

The pinning phenomenon in polymer models has been studied both in the physics and mathematics literature. We refer to $[6,10,11]$ for a mathematical account including also various references in physics. In general, the parameter $\beta$ can have any sign (thus $\beta<0$ 
corresponds to the attractive case with our notations) and in the simplest case of a unique interface at level zero without wall, one observes a localization transition at $\beta=0$. The case $\beta<0$ is known as the repulsive or delocalized phase as the polymer typically touches the interface only finitely many times. The case $\beta>0$ is called instead the attractive or localized phase as the polymer typically touches the interface a positive fraction of time. Caravenna and Pétrélis studied the case of multiple interfaces periodically located at levels $t_{n} \mathbb{Z}$, where the period $t_{n} \in \mathbb{N}$ depends on the size of the polymer $n$, both in the attractive [5] and repulsive case [4]. In contrast, the positions of our interfaces are random, but do not vary in $n$. However, the size of the relevant gaps does depend on $n$, which explains why we use (and extend) some estimates from [4]. Note also the difference between our results, since Caravenna and Pétrélis obtained results at the level of paths, which means information on the (de)localization of the path of the random walk under the polymer measure

$$
\frac{\mathrm{dP}_{n}^{\beta}}{\mathrm{dP}} \propto \exp \left(-\beta \sum_{k=1}^{n} 1_{\left\{S_{k} \in \tau\right\}}\right),
$$

(where $\propto$ means "proportional to") while this is not the purpose of the present paper, see Comment 8 in Section 2.3. Our work can thus be viewed as a natural extension of Caravenna and Pétrélis' model by considering layers with random widths. This direction was actually already suggested in the monograph [6, Chap. 7].

From the physics literature, let us also mention the article [1] where the authors consider a random polymer in a layered environment with two types of solvant.

1.3. Assumption on the obstacles. We now put a probability measure $\mathbb{P}$ on the environment of obstacles. We denote by $T_{k}=\tau_{k}-\tau_{k-1}$, for $k \in \mathbb{N}$, the increments, that is the size of the intervals between two consecutive obstacles, which we call gaps. We assume that, under $\mathbb{P}, \tau$ is a discrete renewal process, that is the $\left(T_{k}\right)$ 's are i.i.d. $\mathbb{N}$-valued random variables. We further assume that $\tau_{0}=0$ and that the increments have a power-tail distribution:

$$
\mathbb{P}\left(T_{1}=n\right) \sim c_{\tau} n^{-(1+\gamma)}, \quad \gamma>0, \quad n \rightarrow \infty,
$$

where $c_{\tau}$ is a positive constant and throughout the paper, $u_{n} \sim v_{n}$ means $u_{n}=[1+o(1)] v_{n}$ as $n \rightarrow \infty$. We recall the following standard limit theorem, see e.g. Petrov [14, Theorem 14, p. 91].

Proposition 1.1. If $\gamma \in(0,1]$, the sequence $\left(\tau_{n} / n^{1 / \gamma}\right)_{n \in \mathbb{N}}$ converges in law to a (totally skewed to the right) $\gamma$-stable random variable with scale parameter $c_{\tau}$. If $\gamma>1$ then $\left(\tau_{n} / n\right)_{n \in \mathbb{N}}$ converges almost-surely to $\mathbb{E}\left(T_{1}\right)$.

1.4. Parabolic Anderson model with a correlated potential. Our model is also connected to a discrete analogue of the parabolic Anderson model (PAM) with potential $V(x)=-\beta 1_{\{x \in \tau\}}-\infty 1_{\{x \leq 0\}}$, that is the heat equation with random potential $V$,

$$
\partial_{t} u=\Delta u+V u \text {. }
$$

There is a rich literature and intense activity around the PAM. We refer to König [13] for a recent survey on this topic. Note that the potential is usually chosen as a sequence of random variables that are independent in the space parameter. In contrast, our potential exhibits long-range spatial correlations, that is one of the research direction suggested in [13, Section 7.2]. Such models have been also considered by physicists. Let us mention for instance the Aubry-André model [3] in which the potential, defined by an irrational rotation, is ergodic but not even mixing. Interestingly this model is known for exhibiting a 
phase transition even in dimension one. For a review of potentials in discrete and continuous space, we refer to [13, Section 1.5].

Let us end this section with a reference to the classical monograph by Sznitman [18] on random motions in random media. Chapter 6 is of particular interest to us as it highlights the link with directed polymers in the presence of columnar defects and introduces the concept of pinning effect of quenched path measures, see again Comment 8 in Section 2.3.

\section{Results}

Before we can state our main theorem, we need Proposition 2.1 below, which gives the existence of an asymptotic cost of crossing per obstacle. Let us define, for $\ell \in \mathbb{N}$, the random variable (with respect to $\tau$ )

$$
\lambda(\ell, \beta)=-\frac{1}{\ell} \log \mathrm{P}^{\beta}\left(H_{\tau_{\ell}}<H_{0} \wedge \sigma\right) .
$$

Note that in the above definition $H_{0}$ could be safely replaced by $H_{\mathbb{Z}^{-}}$.

Proposition 2.1. For all $\beta>0$ there exists a positive constant $\lambda(\beta)=\lambda(\beta, \mathbb{P})$ such that, $\mathbb{P}$-a.s. and in $L_{1}(\mathbb{P})$,

$$
\lim _{\ell \rightarrow \infty} \lambda(\ell, \beta)=\lambda(\beta)
$$

with

$$
0 \leq \lambda(\beta)-\beta \leq \mathbb{E}\left(\log T_{1}\right)+\log 2 .
$$

Note that $\log T_{1}$ is integrable because of (1.8).

From now on we set

$$
N=N(n)=n^{\frac{\gamma}{\gamma+2}}, \quad F_{n}=-\frac{1}{N} \log Z_{n}, \quad n \geq 1 .
$$

We now have all tools and notations in hand to state our main theorem:

Theorem 2.2. The sequence of random variables $\left(F_{n}\right)_{n \in \mathbb{N}}$ converges in $\mathbb{P}$-distribution to the random variable

$$
F:=\inf _{(x, y) \in \Pi}\left\{\lambda(\beta) x+\frac{\pi^{2}}{2 y^{2}}\right\}
$$

where $\Pi$ is a Poisson point process on $\mathbb{R}^{+} \times \mathbb{R}_{*}^{+}$with intensity $p=\mathrm{d} x \otimes c_{\tau} \gamma y^{-(1+\gamma)} \mathrm{d} y$.

With a slight abuse of notation we write $(x, y) \in \Pi$ to mean that $(x, y)$ is in the support of $\Pi$. As a slight but not substantial improvement of this result, we also establish a functional version of this theorem, see Comment 10 in Section 2.3 for a precise statement and Appendix B for its proof.

Before going further, let us first of all give an explanation, at a heuristic level, of the choice of the normalization in (2.4). The argument is of the type one uses to find volume exponents in some polymer models and is sometimes referred to as a Flory argument. We assume that at a large time $n$ the walk has visited at most $N$ obstacles and has remained confined in the largest visible gap, and we find the value of $N$ with the best energy-entropy balance. By basic extreme-value theory, the size of that gap is of order $N^{1 / \gamma}$, and by a standard small-ball estimate (see Proposition 3.1 below for a precise statement) the entropic cost of being confined in that gap for a time interval of length $n$ is of order $n N^{-2 / \gamma}$. Also, the cost of crossing $N$ obstacles is of order $N$, see Proposition 2.1 above. Finally, 
by equating these two costs, one finds the optimal choice $N=n^{\frac{\gamma}{\gamma+2}}$. As a consequence, the walk has travelled a distance of order $n^{\frac{1 \vee \gamma}{\gamma+2}}$ from the origin (see Proposition 1.1). This confinement strategy will be justified during the proof of our theorem. The Poisson point process appears when one tries to make explicit the limit law of the environment after this renormalisation, see Section 2.2.

Outline of the section. We first prove Proposition 2.1 and provide properties of the function $\lambda(\cdot)$ in Section 2.1. Then, in Section 2.2, we consider the environment as a point process and investigate some properties of its limit, which turns out to be the Poisson point process that appears in Theorem 2.2. We conclude with some comments in Section 2.3.

\subsection{Asymptotic cost of crossing obstacles.}

Proof of Proposition 2.1. Let us define a collection of random variables indexed by:

$$
\mathcal{Z}(i, j)=-\log \mathrm{P}_{\tau_{i}}^{\beta}\left(H_{\tau_{j}}<H_{\tau_{i}} \wedge \sigma\right), \quad 0 \leq i<j .
$$

Thus, we are interested in the limit of $(\mathcal{Z}(0, \ell) / \ell)_{\ell \in \mathbb{N}}$. Let $1 \leq i<j<k$. To go from $\tau_{i}$ to $\tau_{k}$ without being killed, one strategy is to go first from $\tau_{i}$ to $\tau_{j}$ and survive, then from $\tau_{j}$ to $\tau_{k}$ and survive without coming back to $\tau_{j}$, which, by the Markov property, leads to the inequality

$$
\mathcal{Z}(i, k) \leq \mathcal{Z}(i, j)+\mathcal{Z}(j, k) .
$$

By stationarity of the sequence $\left(T_{k}\right)_{k \in \mathbb{N}}, \mathcal{Z}(i, j)$ has the same law as $\mathcal{Z}(0, j-i)$. Moreover, by using the standard result

$$
\mathrm{P}_{1}\left(H_{t}<H_{0}\right)=1 / t \quad(t \geq 2)
$$

we obtain

$$
\mathcal{Z}(i-1, i) \leq \beta+\log T_{i}+\log 2, \quad i \in \mathbb{N} .
$$

We get by (2.7) and (2.9)

$$
\beta \ell \leq \mathcal{Z}(0, \ell) \leq(\beta+\log 2) \ell+\sum_{1 \leq i \leq \ell} \log T_{i}
$$

(the lower bound follows simply from the fact that surviving to a newly visited obstacle costs at least $e^{-\beta}$ ). We may now conclude with Kingman's sub-additive ergodic theorem (see Theorem 7.4.1 in [8]) and the law of large numbers.

Proposition 2.3. The function $\beta \rightarrow \lambda(\beta)$ is continuous on $\mathbb{R}_{+}^{*}$.

Proof of Proposition 2.3. We prove that the function is concave on $(0,+\infty)$ : as it is finite, it implies continuity. We observe by integrating out $\mathcal{N}$ that

$$
\lambda(\ell, \beta)=-\frac{1}{\ell} \log \mathrm{E}\left(\exp \left(-\beta \sum_{k=1}^{H_{\tau_{\ell}}} 1_{\left\{S_{k} \in \tau\right\}}\right) 1_{\left\{H_{\tau_{\ell}}<H_{0}\right\}}\right) .
$$

A basic interchange theorem allows us to write

$$
\partial_{\beta}^{2} \lambda(\ell, \beta)=-\frac{1}{\ell} \operatorname{Var}_{\widetilde{\mathrm{P}}_{\beta}}\left(\sum_{k=1}^{H_{\tau_{\ell}}} 1_{\left\{S_{k} \in \tau\right\}}\right) \leq 0,
$$


where $\widetilde{\mathrm{P}}_{\beta}$ is absolutely continuous with respect to $\mathrm{P}$, with Radon-Nikodym derivative:

$$
\frac{\mathrm{d} \widetilde{\mathrm{P}}_{\beta}}{\mathrm{dP}} \propto \exp \left(-\beta \sum_{k=1}^{H_{\tau_{\ell}}} 1_{\left\{S_{k} \in \tau\right\}}\right) 1_{\left\{H_{\tau_{\ell}}<H_{0}\right\}} .
$$

We deduce thereby that $\beta \mapsto \lambda(\ell, \beta)$ is concave. Therefore $\lambda$ is concave as the almost-sure limit of concave functions.

2.2. Convergence of the environment. In this section we recall a few elements from the theory of point processes, see [16] for more background on this topic.

Define the quadrant $E:=[0,+\infty) \times(0,+\infty)$ and consider $\mathcal{E}$ the Borel $\sigma$-algebra on $E$. We say a measure $\mu$ on $(E, \mathcal{E})$ to be a point measure if $\mu$ can be written

$$
\mu:=\sum_{i=1}^{\infty} \delta_{z_{i}}
$$

where, for any $a$ in $E, \delta_{a}$ denotes the Dirac measure in $a$ and $\left(z_{i}\right)_{i \geq 1}$ is a family of points in $E$. If $\mu$ can be written as in (2.14) we say that $z_{i} \in \mu(i \geq 1)$ even if we should say that $z_{i}$ is in the support of $\mu$.

We call $M_{p}(E)$ the set of all Radon point measures on $E$, that are the point measures $\mu$ such that $\mu(K)<\infty$ for all compact sets $K \subset E$. We endow $M_{p}(E)$ with the $\sigma$-algebra $\mathcal{M}_{p}(E)$ defined as the smallest $\sigma$-algebra that makes applications $\mu \mapsto \mu(F)$ measurable for all $F \in \mathcal{E}$. Let $C_{K}^{+}(E)$ be the set of continuous non-negative functions on $E$ with compact support. A sequence $\left(\mu_{n}\right)_{n \geq 1}$ in $M_{p}(E)$ is said to converge vaguely to $\mu$, which we note $\mu_{n} \stackrel{v}{\rightarrow} \mu$, if for any $f$ in $C_{K}^{+}(E)$

$$
\int f \mathrm{~d} \mu_{n} \rightarrow \int f \mathrm{~d} \mu, \quad n \rightarrow \infty .
$$

This provides a topology on $M_{p}(E)$ that turns out to be metrisable, separable, and complete. In this context, a sequence of probability measures $\left(P_{n}\right)_{n \geq 1}$ on $\mathcal{M}_{p}(E)$ converges weakly to $P$, which we note $P_{n} \stackrel{w}{\rightarrow} P$, if for every $\Theta$ vaguely continuous and bounded on $M_{p}(E)$,

$$
\int \Theta \mathrm{d} P_{n} \rightarrow \int \Theta \mathrm{d} P, \quad n \rightarrow \infty
$$

We now come back to our context. For $n \geq 1$ we define

$$
\begin{aligned}
& \left(X_{i}^{n}, Y_{i}^{n}\right):=\left(\frac{i-1}{n}, \frac{T_{i}}{n^{1 / \gamma}}\right) \quad \text { for all } i \geq 1, \\
& \text { and } \quad \Pi_{n}=\sum_{i=1}^{\infty} \delta_{\left(X_{i}^{n}, Y_{i}^{n}\right) .}
\end{aligned}
$$

We observe that $\Pi_{n}$ is a random variable that takes values in $M_{p}(E)$. Recall the definition of $c_{\tau}$ in (1.8) and define $\Pi$ to be a Poisson point process on $E$ with intensity

$$
p:=\mathrm{d} x \otimes \frac{c_{\tau} \gamma}{y^{\gamma+1}} \mathrm{~d} y,
$$

that is, for all finite families of disjoint events $\left(A_{i}\right)_{1 \leq i \leq n} \in \mathcal{E}^{n},\left(\Pi\left(A_{i}\right)\right)_{1 \leq i \leq n}$ are independent Poisson random variables with respective parameters $\left(p\left(A_{i}\right)\right)_{1 \leq i \leq n}$.

Proposition 2.4. It holds that $\Pi_{n} \stackrel{w}{\rightarrow} \Pi$. 
Proof. The proof follows from [16, Proposition 3.21 p. 154] once noticed that for all $y>0$, the sequence $\left(n \mathbb{P}\left(T_{1}>y n^{1 / \gamma}\right)\right)_{n \geq 1}$ converges to $c_{\tau} / y^{\gamma}$ when $n$ goes to infinity.

We define for any $\lambda>0$,

$$
\begin{array}{rlcc}
\psi^{\lambda}: & E & \rightarrow & \mathbb{R}^{+} \\
(x, y) & \mapsto & \lambda x+\frac{\pi^{2}}{2 y^{2}},
\end{array}
$$

and for any $\mu$ in $M_{p}(E)$,

$$
\Psi^{\lambda}(\mu):=\inf _{(x, y) \in \mu} \psi^{\lambda}(x, y)
$$

Let us stress that

$$
F=\inf _{(x, y) \in \Pi}\left\{\lambda(\beta) x+\frac{\pi^{2}}{2 y^{2}}\right\}=\Psi^{\lambda(\beta)}(\Pi) .
$$

We shall write $F^{\beta}$ instead of $F$ when we want to stress the dependence on this parameter.

The rest of the section is devoted to various results relative to the environment $\Pi$.

Proposition 2.5. For all $\lambda>0, \Psi^{\lambda}(\Pi)$ is almost surely well defined and positive. Moreover the infimum in the definition of $\Psi^{\lambda}$ is almost surely achieved at a unique point $\left(x^{*}, y^{*}\right)$ in E.

Proof of Proposition 2.5. As there is almost surely at least one point in $\Pi$, the infimum is well defined. Moreover, there are almost surely finitely many points in $[0,1] \times[1,+\infty)$. We define $\bar{Y}$ as the maximal second coordinate among these points if $[0,1] \times[1,+\infty) \cap \Pi$ is not empty, otherwise we set $\bar{Y}=1$. Note that $\bar{Y}$ is almost surely finite and $\psi^{\lambda}(x, y) \geq$ $\min \left\{\pi^{2} /\left(2 \bar{Y}^{2}\right), \lambda\right\}$ for any $(x, y) \in \Pi$, which proves that $\Psi^{\lambda}(\Pi)$ is almost surely positive.

For $\lambda>0$ and $u>0$, we introduce the set

$$
A_{u}^{\lambda}:=\left\{(x, y) \in E \text { such that } \psi^{\lambda}(x, y) \leq u\right\},
$$

and note that $\Pi\left(A_{u}^{\lambda}\right)<\infty$ almost surely.

Let us denote by $C$ the event that the minimum in the definition of $\Psi$ is achieved. We observe that for any $u>0$,

$$
P(\Psi(\Pi)<u)=P\left(\Psi(\Pi)<u, \Pi\left(A_{u}^{\lambda}\right)<\infty\right) \leq P(\Psi(\Pi)<u, \Pi \in C) .
$$

As $\lim _{u \rightarrow+\infty} P(\Psi(\Pi)<u)=1$ and $\lim _{u \rightarrow+\infty} P(\Psi(\Pi)<u, \Pi \in C)=P(\Pi \in C)$, we obtain that $P(\Pi \in C)=1$.

It remains to prove that the infimum is almost surely achieved at only one point. For $u>0$, let $D_{u}$ be the event that two points of $\Pi \cap A_{u}^{\lambda}$ have the same image by $\psi^{\lambda}$ and $D$ be the event that two points of $\Pi$ have the same image by $\psi^{\lambda}$. For $n \in \mathbb{N}$ and conditional on $\left\{\Pi\left(A_{u}^{\lambda}\right)=n\right\}$, the restriction of $\Pi$ to $A_{u}^{\lambda}$ has the same law as $\sum_{i=1}^{n} \delta_{X_{i}}$, where the $\left(X_{i}\right)_{1 \leq i \leq n}$ 's are i.i.d. with continuous law $p$ restricted to $A_{u}^{\lambda}$ and renormalised to a probability measure. This implies that $P\left(\Pi \in D_{u} \mid \Pi\left(A_{u}^{\lambda}\right)\right)=0$ and thus $P\left(\Pi \in D_{u}\right)=0$. We obtain

$$
P(\Psi(\Pi)<u)=P\left(\Psi(\Pi)<u, \Pi \in D_{u}^{c}\right) .
$$

The first term converges to 1 while the second one converges to $P(\Pi \in D)$ when $u$ goes to infinity. This proves $P(\Pi \in D)=1$ and concludes the proof. 
Unfortunately, it is difficult to work directly with $\Psi^{\lambda}$ as it appears not to be a vaguely continuous function on $M_{p}(E)$. For this reason, we introduce the function $\Psi_{K}^{\lambda}$, for any compact set $K \subset E$, defined by

$$
\Psi_{K}^{\lambda}(\mu):=\inf _{(x, y) \in \mu \cap K} \psi^{\lambda}(x, y), \quad \mu \in M_{p}(E) .
$$

The advantage of restricting the infimum to a compact set lies in the following lemma.

Lemma 2.6. For any compact set $K \subset E$ and $\lambda>0$, the function $\Psi_{K}^{\lambda}$ is vaguely continuous on $M_{p}(E)$.

Proof. Consider a sequence $\left(\mu_{n}\right)_{n \geq 1}$ in $M_{p}(E)$ that converges vaguely to $\mu$. Due to Proposition 3.14 in [16], $\mu \in M_{p}(E)$. Suppose $\mu(K)=k$, that is $\mu(\cdot \cap K)$ writes $\sum_{i=1}^{k} \delta_{\left(x_{i}, y_{i}\right)}$ where $\left(x_{i}, y_{i}\right)_{1 \leq i \leq k}$ is a family in $K$. By Proposition 3.13 in [16] there exists for all $n$ larger than some $n(K)$ a family $\left(x_{i}^{n}, y_{i}^{n}\right)_{1 \leq i \leq k}$ such that $\mu_{n}(\cdot \cap K)=\sum_{i=1}^{k} \delta_{\left(x_{i}^{n}, y_{i}^{n}\right)}$. Moreover, for all $1 \leq i \leq k$, the sequence $\left(x_{i}^{n}, y_{i}^{n}\right)_{n \geq 1}$ converges to $\left(x_{i}, y_{i}\right)$ as $n$ goes to infinity. This implies that $\Psi_{K}^{\lambda}\left(\mu_{n}\right)$ converges to $\Psi_{K}^{\lambda}(\mu)$.

We conclude this section with the following technical lemma:

Lemma 2.7. The family $\left(F^{\beta-\varepsilon}\right)_{\varepsilon \geq 0}$ (seen as functions of the random measure $\Pi$ ) converges non-decreasingly to $F^{\beta}$ when $\varepsilon \rightarrow 0$, almost surely.

Proof. Using Proposition 2.5 we can define almost surely a random point $\left(X^{*}(\beta), Y^{*}(\beta)\right)$ such that

$$
\psi^{\lambda(\beta)}\left(X^{*}(\beta), Y^{*}(\beta)\right)=\Psi^{\lambda(\beta)}(\Pi) .
$$

We first prove that $\left(X^{*}(\beta-\varepsilon), Y^{*}(\beta-\varepsilon)\right)=\left(X^{*}(\beta), Y^{*}(\beta)\right)$ almost surely if $\varepsilon>0$ is chosen small enough. Let $\varepsilon_{0} \in(0, \beta)$. Consider some $x \geq x_{0}:=\frac{2 \Psi^{\lambda(\beta)}(\Pi)}{\lambda\left(\beta-\varepsilon_{0}\right)}$ and any $y>0$. As $\lambda(\cdot)$ and $\Psi^{\lambda(\cdot)}$ are non-decreasing functions of $\beta$ it holds that

$$
\psi^{\lambda(\beta-\varepsilon)}(x, y) \geq \lambda(\beta-\varepsilon) x \geq 2 \Psi^{\lambda(\beta-\varepsilon)}(\Pi)
$$

for all $\varepsilon<\varepsilon_{0}$, and we conclude that $X^{*}(\beta-\varepsilon)<x_{0}$ for any $\varepsilon<\varepsilon_{0}$.

Consider now some $y \leq y_{0}:=\frac{\pi}{2 \sqrt{\Psi^{\lambda(\beta)}(\Pi)}}$ and any $x>0$. We thus obtain

$$
\psi^{\lambda(\beta-\varepsilon)}(x, y) \geq \frac{\pi^{2}}{2 y^{2}} \geq 2 \Psi^{\lambda(\beta-\varepsilon)}(\Pi),
$$

and we conclude that $Y^{*}(\beta-\varepsilon)>y_{0}$ for any $\varepsilon<\varepsilon_{0}$. We deduce thereof that almost surely

$$
F^{\beta-\varepsilon}=\inf _{(x, y) \in \Pi \cap\left\{x<x_{0}, y>y_{0}\right\}} \psi^{\lambda(\beta-\varepsilon)}(x, y) .
$$

Finally, observe that $\Pi\left(x<x_{0}, y>y_{0}\right)<\infty$ almost surely, so that there are only finitely many candidates for $\left(X^{*}(\beta-\varepsilon), Y^{*}(\beta-\varepsilon)\right)$. Pick $(X, Y) \in \Pi \cap\left\{x<x_{0}, y>y_{0}\right\}$ that is not $\left(X^{*}(\beta), Y^{*}(\beta)\right)$ (if there is no such point there is nothing more to prove as $\left(X^{*}(\beta), Y^{*}(\beta)\right)$ is then the only candidate). The function $\varepsilon \mapsto \lambda(\beta-\varepsilon) X+\frac{\pi^{2}}{2 Y^{2}}$ is (i) strictly larger than $\lambda(\beta) X^{*}(\beta)+\frac{\pi^{2}}{2\left(Y^{*}(\beta)\right)^{2}}$ at $\varepsilon=0$ due to Proposition 2.5 and (ii) continuous due to Proposition 2.3. Therefore,

$$
\lambda(\beta-\varepsilon) X+\frac{\pi^{2}}{2 Y^{2}}>\lambda(\beta) X^{*}(\beta)+\frac{\pi^{2}}{2\left(Y^{*}(\beta)\right)^{2}}
$$


for $\varepsilon>0$ small enough. As $\Pi\left(\left\{x<x_{0}, y>y_{0}\right\}\right)<\infty$ almost surely, we can choose $\varepsilon$ small enough so that the last inequality holds for all points in $\Pi \cap\left\{x<x_{0}, y>y_{0}\right\}$ different from $\left(X^{*}(\beta), Y^{*}(\beta)\right)$. Therefore, we may from now on consider $\varepsilon>0$ small enough so that $\left(X^{*}(\beta-\varepsilon), Y^{*}(\beta-\varepsilon)\right)=\left(X^{*}(\beta), Y^{*}(\beta)\right)$.

From what precedes, we get

$$
\left|\Psi^{\lambda(\beta-\varepsilon)}(\Pi)-\Psi^{\lambda(\beta)}(\Pi)\right| \leq|\lambda(\beta-\varepsilon)-\lambda(\beta)| X^{*}(\beta) .
$$

Again, as $\lambda$ is continuous, $\Psi^{\lambda(\beta-\varepsilon)}(\Pi)$ converges almost surely to $\Psi^{\lambda(\beta)}(\Pi)$ when $\varepsilon$ goes to 0 . Finally,

$$
F^{\beta-\varepsilon} \underset{\varepsilon \rightarrow 0}{\rightarrow} F^{\beta} \quad \text { a.s. }
$$

Moreover, as $\lambda$ is non-decreasing with $\beta$, the convergence is monotone:

$$
F^{\beta-\varepsilon} \nearrow_{\varepsilon \rightarrow 0} F^{\beta} \quad \text { a.s. }
$$

2.3. Comments. We collect here a number of comments and remarks about our result.

1. We do not consider the annealed survival probability, which decreases at most polynomially fast since

$$
\mathbb{E} \mathrm{P}\left(\sigma \wedge H_{\mathbb{Z}^{-}}>n\right) \geq \frac{1}{2} \mathrm{P}_{1}\left(H_{0} \geq n\right) \mathbb{P}\left(\tau_{1}>n\right) \sim \frac{1}{2} c_{\tau} n^{-\gamma-\frac{1}{2}}
$$

as $n \rightarrow \infty$, and thus has a completely different behaviour than the quenched probability.

2. Note that we cannot hope for better than weak convergence. Indeed, if $F_{n}$ would converge to $F$ almost-surely, then $F$ would be measurable with respect to the tail $\sigma$-algebra of the family $\left(T_{i}\right)_{i \in \mathbb{N}}$. As the increments are independent, the latter is trivial due to the $0-1$ law, and $F$ would be deterministic.

3. In the case $\gamma \leq 1$, the variational formula in (2.5) has an alternative representation in terms of a subordinator, which reads

$$
F=\inf _{t \geq 0}\left\{\lambda(\beta) t+\frac{\pi^{2}}{2\left(\Delta \mathcal{S}_{t}\right)^{2}}\right\}
$$

where $\left(\mathcal{S}_{t}\right)_{t \geq 0}$ is a $\gamma$-stable subordinator and $\Delta \mathcal{S}_{t}=\mathcal{S}_{t}-\mathcal{S}_{t}^{-}=\mathcal{S}_{t}-\lim _{u \rightarrow t^{-}} \mathcal{S}_{u}$.

4. We can compute explicitly the tail distribution function of the limiting law $F=\Psi^{\lambda(\beta)}(\Pi)$ in Theorem 2.2. Recall (2.18), (2.19) and (2.22). For any $u \geq 0$ (we write $\lambda$ instead of $\lambda(\beta)$ to lighten notations),

$$
\mathbb{P}(F \geq u)=\mathbb{P}\left(\Pi\left(A_{u}^{\lambda}\right)=0\right)=\exp \left(-p\left(A_{u}^{\lambda}\right)\right) .
$$

Since $A_{u}^{\lambda}=\left\{(x, y): 0 \leq x<u / \lambda, y \geq \frac{\pi}{\sqrt{2(u-\lambda x)}}\right\}$, we get by a straightforward computation that

$$
\mathbb{P}(F \geq u)=\exp \left(-\frac{c_{\tau}}{\lambda(\beta) \pi^{\gamma}(\gamma+2)}(2 u)^{\frac{\gamma}{2}+1}\right), \quad u \geq 0
$$

5. The case $\gamma=0$ is left open. In this case, a gap distribution of the form (1.8) is no longer appropriate and one should instead assume that $\mathbb{P}\left(T_{1}=n\right) \sim L(n) / n$, where $L$ is a non-negative slowly varying function such that $\sum L(n) / n$ is finite. Complications may 
arise at two levels: (i) the normalization of $\max _{1 \leq i \leq n} T_{i}$, that we use to guess the value of $N$, and (ii) the integrability of $\log T_{1}$, that we use in Proposition 2.1. For instance, if $L(n)=(\log n)^{-2}$ then $\mathbb{E}\left(\log T_{1}\right)=\infty$ and $\max _{1 \leq i \leq n} T_{i}$ has a completely different type of renormalization since, as one can readily show, $(1 / \sqrt{n}) \log \max _{1 \leq i \leq n} T_{i}$ converges to a non-trivial probability law with cumulative distribution function $x \mapsto \exp \left(-x^{-2}\right) 1_{\{x>0\}}$, as $n \rightarrow \infty$.

6. We state without proof an alternative expression for $\lambda(\beta)$ based on ergodic theory considerations. To this end, let $\widetilde{\tau}$ be an independent copy of $\tau$, as defined in Section 1.3. We write $\widetilde{\mathbb{E}}$ for the expectation with respect to $\widetilde{\tau}$. Suppose that the random walk is now free to visit $\mathbb{Z}^{-}$but is killed by the set $-\widetilde{\tau}$ (note the minus sign), with the same probability $1-\exp (-\beta)$, and denote by $\widetilde{\sigma}$ the corresponding killing time. Then,

$$
\lambda(\beta)=-\mathbb{E} \widetilde{\mathbb{E}} \log \mathrm{P}^{\beta}\left(H_{\tau_{1}}<\widetilde{\sigma}\right) .
$$

Assuming this last equality, we could readily prove using the dominated convergence theorem that $\lambda$ is also continuous at 0 .

7. Equation (2.3) does not give much information about the behaviour of $\lambda(\beta)$ at 0 , that remains an open question. We expect however that $\beta=o(\lambda(\beta))$ as $\beta \rightarrow 0$ and we now explain why. To this end, recall (2.37) and the related notations above. By integrating out $\mathcal{N}$ and differentiating in $\beta$ we obtain

$$
\lim _{\beta \rightarrow 0} \lambda^{\prime}(\beta)=\widetilde{\mathbb{E}} \mathbb{E} \mathrm{E}\left(\sum_{k=1}^{H_{\tau_{1}}} 1_{\left\{S_{k} \in-\widetilde{\tau}\right\}}\right),
$$

that we expect to be infinite. Indeed, by first restricting the walk to make its first step to the left and then using the symmetry of the random walk,

$$
\widetilde{\mathbb{E}} \mathbb{E} \mathrm{E}\left(\sum_{k=1}^{H_{\tau_{1}}} 1_{\left\{S_{k} \in-\widetilde{\tau}\right\}}\right) \geq \frac{1}{2} \widetilde{\mathbb{E}} \mathrm{E}_{-1}\left(\sum_{k=1}^{H_{0}} 1_{\left\{S_{k} \in-\widetilde{\tau}\right\}}\right)=\frac{1}{2} \widetilde{\mathbb{E}} \mathrm{E}_{1}\left(\sum_{k=1}^{H_{0}} 1_{\left\{S_{k} \in \widetilde{\tau}\right\}}\right) .
$$

We now interchange integrals and use the renewal theorem to obtain, at least for $\gamma \neq 1$,

$$
\lim _{\beta \rightarrow 0} \lambda^{\prime}(\beta) \geq \frac{1}{2} \mathrm{E}_{1}\left(\sum_{k=1}^{H_{0}} \widetilde{\mathbb{P}}\left(S_{k} \in \widetilde{\tau}\right)\right) \geq \frac{C}{2} \mathrm{E}_{1}\left(\sum_{k=1}^{H_{0}}\left(1+S_{k}\right)^{(\gamma-1) \wedge 0}\right) .
$$

Since, by Ray-Knight's theorem, the mean number of visits to $x \in \mathbb{N}_{0}$ between time 1 and $H_{0}$ equals 1 under $\mathrm{P}_{1}$, we get

$$
\lim _{\beta \rightarrow 0} \lambda^{\prime}(\beta) \geq C \sum_{x \in \mathbb{N}_{0}}(1+x)^{(\gamma-1) \wedge 0}=\infty .
$$

8. Note that we offer no path statement. In other words, we do not prove anything about the behaviour of the walk conditioned to survive for a long time $n$. However, as it is often the case with this type of model, our result and the method of proof suggest a path strategy, which in our context corresponds to a confinement (or localization) strategy. To be more precise, we roughly expect that as $n$ is large, the walk reaches the obstacle labelled $X^{*}(\beta) N$ and then remains in the corresponding gap, of size $Y^{*}(\beta) N^{1 / \gamma}$, where $\left(X^{*}(\beta), Y^{*}(\beta)\right)$ is distributed as the unique minimizer of the random variational problem in Theorem 2.2. In other words, the path (or polymer) gets stuck in a slab which is large enough and not too far from the origin. Surprisingly, the repulsive interaction between the path and the obstacles 
leads to a pinning effect on the quenched path measures, as explained by Sznitman [18, Chapter 6]. Proving such a result should demand substantial additional work, as one would most likely need sharper bounds on the survival probability (partition function) and fine controls on the ratio of survival probabilities restricted to suitable events. Nevertheless, this can be considered as an interesting direction of research. Let us mention that Ding and $\mathrm{Xu}$ [7] recently obtained a path confinement result in the case of quenched hard Bernoulli obstacles for $d \geq 2$.

9. Let us stress that the scaling $t^{\gamma /(\gamma+2)}$ that appears in our Theorem 2.2 is different from the scaling of the PAM in a bounded i.i.d. potential. In this case [13, Example 5.10] states that the correct scaling is $t$ up to a logarithmic correction. Hence we are in a case where the correlations of the potential have a drastic effect on the asymptotic behaviour of the survival probability.

10. With a few additional efforts, we can actually upgrade Theorem 2.2 to a functional version. Define, for $n \geq 1$, the random process $\left(F_{n}(s)\right)_{s \geq 0}$ as the linear interpolation of $s \in \frac{\mathbb{N}}{n} \mapsto-\frac{1}{N} \log Z_{n s}$.

Theorem 2.8. Let $T>0$. Under $\mathbb{P}$, the sequence of random processes $\left(F_{n}(s)\right)_{s \in[0, T]}, n \in \mathbb{N}$, converges in law, in the space of continuous functions on $[0, T]$ endowed with the uniform topology, to the random process

$$
F(s):=\inf _{(x, y) \in \Pi}\left\{\lambda(\beta) x+\frac{\pi^{2} s}{2 y^{2}}\right\}, \quad s \in[0, T] .
$$

Let us note that, even if this result is stronger than Theorem 2.2, we learn only little from its proof, that requires only minor modifications with respect to that of Theorem 2.2. That is why we choose to expose Theorem 2.2 as our main result and defer the proof of Theorem 2.8 to Appendix B.

Organisation of the paper. The rest of the article is devoted to the proof of Theorem 2.2. The general strategy is the following. In Section 3 we first establish several results for the killed random walk that are valid for any deterministic sequence of obstacles. Let us mention in particular the existence of a limiting survival rate for a periodic sequence of obstacles, which is interesting by itself (see Proposition 3.3). Then, we introduce the notion of good environments, that are environments for which we can provide suitable bounds on the survival probability $Z_{n}$, see (3.71). In Sections 4 and 5 we successively prove lower and upper bounds, assuming only that the environment is good. We complete the proof in Section 6: first we show that we can adjust parameters so that environments are asymptotically good, with a probability arbitrarily close to one; then we use the two bounds obtained in the previous sections to relate the quantity of interest to an explicit functional $\Psi$ of the properly renormalized environment, see (2.20). Finally we let $n$ tend to infinity to prove that $F$ has the same law as $\Psi$ applied to the limiting environment, that is a Poisson point process (see Proposition 2.4).

\section{KEY TOOLS}

In this section we introduce several tools which we will use to prove our result. For convenience, various notations are gathered together in Section 3.1, to which the read may refer back. In Section 3.2 we remind the reader of the so-called small ball estimate and 
establish a rough upper bound on the probability that the walker stays confined in a fixed gap until time $n$, see Proposition 3.4. Section 3.3 contains Proposition 3.6, which states that a walk conditioned to hit a given point $x$ before coming back to 0 does it faster when it is also conditioned on survival until its first visit to $x$. In Section 3.4 we state the two technical Lemmas 3.7 and 3.8 that we will use in Section 5 while proving the upper bound on $Z_{n}$. Finally we introduce the key notion of good environment in Section 3.5. Informally, good environments are those for which we are able to efficiently bound $Z_{n}$. We thus give a list of events, see (3.71), that are essentially the technical conditions we will need in Proposition 5.1 and 4.1 .

3.1. Notations. Let us introduce notations that will be necessary in what comes next.

Killing clock. We recall the definition of the $\mathbb{N}$-valued geometric random variable $\mathcal{N}$ with success parameter $1-e^{-\beta}$ that plays the role of the killing clock.

Records. As we already hinted in the heuristics, only the largest gaps matter. To be more precise, a particular attention is given to record values of the sequence $\left(T_{\ell}\right)$. Therefore, for any increasing sequence of integers $\left(\tau_{i}\right)_{i \in \mathbb{N}_{0}}$ with $\tau_{0}=0$, and defining $t_{i}=\tau_{i}-\tau_{i-1}$ for all $i \in \mathbb{N}$, we let

$$
i(0)=0, \quad i(k)=\inf \left\{i>i(k-1): t_{i+1}>t_{i(k-1)+1}\right\}, \quad k \in \mathbb{N},
$$

be the sequence of record indexes, while

$$
\tau_{k}^{*}=\tau_{i(k)} \quad \text { and } \quad t_{k}^{*}=t_{i(k)+1}, \quad k \in \mathbb{N}_{0} .
$$

We also define

$$
R(a, b)=\{k \in \mathbb{N}: a \leq i(k) \leq b\}, \quad \mathcal{R}(a, b)=i(R(a, b)), \quad a, b \in \mathbb{N}, a<b,
$$

and

$$
R_{\varepsilon}(n)=R\left(\varepsilon N, \varepsilon^{-1} N\right), \quad \mathcal{R}_{\varepsilon}(n)=\mathcal{R}\left(\varepsilon N, \varepsilon^{-1} N\right), \quad n \in \mathbb{N}, \quad \varepsilon>0 .
$$

Finally we write

$$
\mathcal{R}=\mathcal{R}(1, \infty),
$$

for the set of all records. Later in the paper we will apply these notations to the random sequence $\left(T_{\ell}\right)$, in which case $t_{k}^{*}$ writes $T_{k}^{*}$ and all quantities introduced above become random.

Auxiliary random walk. We recall that the clock process $\left(\theta_{n}\right)_{n \in \mathbb{N}}$ is defined by

$$
\theta_{0}=0, \quad \theta_{n+1}=\inf \left\{k>\theta_{n}: S_{k} \in \tau\right\}, \quad n \in \mathbb{N}_{0} .
$$

The process that chronologically keeps track of the obstacles visited by the walk will be denoted by $X=\left(X_{n}\right)_{n \in \mathbb{N}_{0}}$ and is uniquely determined by $\tau_{X_{n}}=S_{\theta_{n}}$. It is not difficult to see that $X$ is a Markov chain on $\mathbb{N}_{0}$, the hitting times of which are denoted by

$$
\zeta_{x}=\inf \left\{n \in \mathbb{N}: X_{n}=x\right\}, \quad x \in \mathbb{N}_{0},
$$

and

$$
\zeta_{k}^{*}=\inf \left\{n \in \mathbb{N}: X_{n}=i(k)\right\}, \quad k \in \mathbb{N}_{0} .
$$

Transition matrices and their moment-generating functions. Let us define

$$
q_{i j}(n)=\mathrm{P}_{\tau_{i}}\left(S_{k} \notin \tau, 1 \leq k<n, S_{n}=\tau_{j}\right), \quad i, j \in \mathbb{N}_{0}, \quad n \in \mathbb{N},
$$


and the associated family of matrices $\{Q(\phi)\}_{\phi \geq 0}$ by

$$
Q_{i j}(\phi)=\sum_{n \in \mathbb{N}} e^{\phi n} q_{i j}(n)=\mathrm{E}_{\tau_{i}}\left(e^{\phi \theta_{1}} 1_{\left\{S_{\theta_{1}}=\tau_{j}\right\}}\right), \quad i, j \in \mathbb{N}_{0}, \phi \geq 0 .
$$

Note that the matrix $\left\{Q_{i j}(0)\right\}_{i, j \in \mathbb{N}_{0}}$ is nothing but the transition matrix of the Markov chain $X$ defined above. These quantities will appear in Lemma 3.7 below and are zero as soon as $|j-i|>1$. Finally, we will also use the following notations for the gap associated to an non-oriented edge $\{i, j\}$ with $|j-i| \leq 1$ :

$$
t_{i j}= \begin{cases}t_{i+1} & \text { if } j=i+1 \\ t_{i} & \text { if } j=i-1, \\ t_{i+1} \vee t_{i} & \text { if } \quad i=j\end{cases}
$$

where $\left(t_{i}\right)$ is a sequence of integers.

3.2. Confinement estimates. One of the key standard estimates in our arguments are the so-called small-ball estimates, that control the probability that a simple random walk stays confined in an interval:

Proposition 3.1. There exist $\mathcal{T}_{0}, c_{1}, c_{2}, c_{3}, c_{4}>0$ such that for all $t>\mathcal{T}_{0}$, the following inequalities hold for all $n \in \mathbb{N}$ such that $n \in 2 \mathbb{N}$ or $n-t \in 2 \mathbb{N}$ :

$$
\begin{gathered}
\frac{c_{1}}{t \wedge n^{1 / 2}} e^{-g(t) n} \leq \mathrm{P}\left(H_{t} \wedge H_{0} \wedge H_{-t}>n\right) \leq \frac{c_{2}}{t \wedge n^{1 / 2}} e^{-g(t) n} \\
\frac{c_{3}}{t^{3} \wedge n^{3 / 2}} e^{-g(t) n} \leq \mathrm{P}\left(H_{t} \wedge H_{0} \wedge H_{-t}=n\right) \leq \frac{c_{4}}{t^{3} \wedge n^{3 / 2}} e^{-g(t) n},
\end{gathered}
$$

where

$$
g(t)=-\log \cos \left(\frac{\pi}{t}\right)=\frac{\pi^{2}}{2 t^{2}}+O\left(\frac{1}{t^{4}}\right), \quad t \rightarrow+\infty .
$$

This proposition is taken from Lemma 2.1 in Caravenna and Pétrélis [4]. A crucial point here is the uniformity of the constants, which gives the uniformity of the constant $C$ in Proposition 3.4.

Caravenna and Pétrélis [4] treated the case of equally spaced obstacles, which we refer to as the homogeneous case, in the sense that increments of $\tau$ are all equal. We summarize their results here.

Proposition 3.2 (Homogeneous case, see Eq. (2.1)-(2.3) in [4]). Let $t \in \mathbb{N}$ and $\tau=t \mathbb{Z}$. There exists a constant $\phi(\beta, t)$ such that

$$
\phi(\beta, t)=-\lim _{n \rightarrow \infty} \frac{1}{n} \log \mathrm{P}(\sigma>n),
$$

with

$$
\phi(\beta, t)=\frac{\pi^{2}}{2 t^{2}}\left(1-\frac{4}{e^{\beta}-1} \frac{1}{t}+o\left(\frac{1}{t}\right)\right) .
$$

Moreover, it is the only solution of the equation:

$$
\left.\mathrm{E}\left(\exp \left(\phi \inf \left\{n \in \mathbb{N}: S_{n} \in \tau\right\}\right)\right)\right)=\exp (\beta), \quad \beta \geq 0 .
$$

Note that the first order term in the expansion of $\phi$ does not depend on $\beta$. It turns out that we are able to extend (3.15), at the price of additional technical work, to deal with the weakly-inhomogeneous case, that is when increments of $\tau$ follow a periodic pattern. We obtain the following: 
Proposition 3.3 (Weakly-inhomogeneous case). Let $p \geq 2, t_{1}, \ldots, t_{p}$ positive integers and $\tau$ be the periodic set $\left\{\tau_{i}: 0 \leq i<p\right\}+\tau_{p} \mathbb{Z}$, where $\tau_{0}=0$ and $\tau_{i}=\sum_{1 \leq j \leq i} t_{j}$ for all $0<i \leq p$. There exists a constant $\phi=\phi\left(\beta ; t_{1}, \ldots, t_{p}\right)$ such that

$$
\phi\left(\beta ; t_{1}, \ldots, t_{p}\right)=-\lim _{n \rightarrow \infty} \frac{1}{n} \log \mathrm{P}(\sigma>n) .
$$

Moreover,

$$
\mathrm{P}(\sigma>n) \leq C n^{2} p \exp \left(-\phi\left(\beta ; t_{1}, \ldots, t_{p}\right) n\right), \quad n \geq 1,
$$

and

$$
\phi\left(\beta, t_{\max }\right) \leq \phi\left(\beta ; t_{1}, \ldots, t_{p}\right)<g\left(t_{\max }\right), \quad t_{\max }=\max _{1 \leq i \leq p} t_{i} .
$$

The proof is deferred to Appendix A. Remark that both inequalities in (3.20) are intuitive: the first one asserts that it is easier to survive in a homogeneous environment with gap $t_{\max }$ than in the original environment. The second one states that one of the strategy to survive is to stay confined in the largest gap. With this estimate in hand, we get our first building block, that is an upper bound on the probability to survive in-between two obstacles, for a general environment $\tau$.

Proposition 3.4. Let $\left(\tau_{i}\right)_{i \in \mathbb{N}_{0}}$ be an increasing sequence of integers with $\tau_{0}=0$ and let $t_{i}=\tau_{i}-\tau_{i-1}$ for all $i \in \mathbb{N}$. There exists a constant $C>0$ such that for all $0 \leq k<r<\ell$, one has

$$
\mathrm{P}_{\tau_{r}}\left(\sigma \wedge H_{\tau_{k}} \wedge H_{\tau_{\ell}}>n\right) \leq C n^{2}(\ell-k) \exp \left(-\phi\left(\beta ; \max \left\{t_{i}: k<i \leq \ell\right\}\right) n\right),
$$

where $\phi(\beta ; \cdot)$ is defined in Proposition 3.2.

Proof of Proposition 3.4. The proof relies on periodization. Since the random walk does not leave the interval $\left(\tau_{k}, \tau_{\ell}\right)$ on the event considered, we may as well replace the renewal $\tau$ by a periodized version, and by translation invariance, consider that the random walk starts at zero. Finally, everything is as if the walk evolves in a new environment $\widetilde{\tau}$, with periodic increments, defined by

$$
\widetilde{\tau}=\left\{\tau_{i}: k \leq i \leq \ell\right\}-\tau_{r}+\left(\tau_{\ell}-\tau_{k}\right) \mathbb{Z},
$$

and we now have to bound from above $\mathrm{P}^{\widetilde{\tau}}\left(\sigma \wedge H_{\widetilde{\tau}_{k-r}} \wedge H_{\widetilde{\tau}_{\ell-r}}>n\right)$, where we put a superscript on $\mathrm{P}$ to stress that the walk evolves among $\widetilde{\tau}$. This probability is certainly smaller than $\mathrm{P}_{0}^{\widetilde{\tau}}(\sigma>n)$, and we may now conclude thanks to Proposition 3.3.

In the following Proposition 3.4 appears to be one of the key estimates in our proof, see (5.11) and (5.8). It is actually possible to obtain this proposition (with a different polynomial correction) without (3.18). We propose hereafter an alternative proof by comparing the survival probability in the weakly-inhomogeneous environment considered in Proposition 3.3 with the same probability in a periodic environment where all gaps have size $t_{\max }=\max _{1 \leq i \leq p} t_{i}$. Yet we choose to keep Proposition 3.3 as it gives a stronger result and is interesting by itself.

Proposition 3.5. We consider the same notations and environment as in Proposition 3.3. Define moreover $\tau^{(\max )}=t_{\max } \mathbb{Z}$. For all $n \in \mathbb{N}_{0}$, for all $\beta \geq 0$,

$$
\mathrm{P}^{\tau}(\sigma>n) \leq \mathrm{P}^{\tau^{(\max )}}(\sigma>n) .
$$

As a consequence, there exists a constant $C>0$ such that for all $n \in \mathbb{N}_{0}$ and $t_{1}, \ldots, t_{p} \in \mathbb{N}$,

$$
\mathrm{P}^{\tau}(\sigma>n) \leq C t_{\max }^{3} \exp \left(-\phi\left(\beta, t_{\max }\right) n\right) .
$$


Proof of Proposition 3.5. Let us start with (3.23). Recall (1.1) and the definition of $X$ above (3.7). From (1.2), it is enough to prove that for all $k \in \mathbb{N}$,

$$
\mathrm{P}^{\tau}\left(\theta_{k} \geq \ell\right) \leq \mathrm{P}_{0}^{\tau^{(\max )}}\left(\theta_{k} \geq \ell\right), \quad \ell \in \mathbb{N}_{0},
$$

which we prove by induction on $k$. To that end, note that, by a standard coupling argument,

$$
\sup _{x \in \tau} \mathrm{P}_{x}^{\tau}\left(\theta_{1} \geq \ell\right) \leq \mathrm{P}_{0}^{\tau^{(\max )}}\left(\theta_{1} \geq \ell\right), \quad \ell \in \mathbb{N}_{0}
$$

The inequality above serves both as the initialization step and in the induction step in the proof of (3.25). Indeed, suppose that (3.25) is true for some $k \in \mathbb{N}$ (for all $\ell \in \mathbb{N}_{0}$ ). Let us prove that it also holds for $k+1$. By applying the strong Markov property at time $\theta_{k}$,

$$
\mathrm{P}^{\tau}\left(\theta_{k+1} \geq \ell\right)=\mathrm{E}^{\tau}\left(u\left(\ell-\theta_{k}, \tau_{X_{k}}\right)\right),
$$

where

$$
u(m, y)=\mathrm{P}_{y}^{\tau}\left(\theta_{1} \geq m\right) 1_{\left\{m \in \mathbb{N}_{0}\right\}}, \quad y \in \tau .
$$

By using (3.26), we obtain

$$
\mathrm{P}^{\tau}\left(\theta_{k+1} \geq \ell\right) \leq \mathrm{E}^{\tau}\left(\widetilde{u}\left(\ell-\theta_{k}\right)\right),
$$

where

$$
\widetilde{u}(m)=\mathrm{P}^{\tau^{(\max )}}\left(\theta_{1} \geq m\right) 1_{\left\{m \in \mathbb{N}_{0}\right\}},
$$

Finally, by using the induction hypothesis, we get, for all $\ell \in \mathbb{N}_{0}$,

$$
\mathrm{E}^{\tau}\left(\widetilde{u}\left(\ell-\theta_{k}\right)\right) \leq \mathrm{E}^{\tau^{(\max )}}\left(\widetilde{u}\left(\ell-\theta_{k}\right)\right)=\mathrm{P}^{\tau^{(\max )}}\left(\theta_{k+1} \geq \ell\right) .
$$

To obtain (3.24), we combine the renewal representation of the survival probability, see [5, Equation (2.11)] with the expansion of $\phi$ in (3.16).

3.3. A decoupling inequality. The next building block is a control on the probability that the walk reaches a given point before a certain fixed time, conditional on survival and not coming back to 0 . In essence, the following proposition tells us that the walk reaches this point stochastically faster in the presence of obstacles:

Proposition 3.6. Let $\left(\tau_{i}\right)_{i \in \mathbb{N}_{0}}$ be an increasing sequence of integers with $\tau_{0}=0$. For all $\beta>0, x \in \mathbb{N}$ and $n \in \mathbb{N}$,

$$
\mathrm{P}^{\beta}\left(H_{x} \leq n \mid \sigma \wedge H_{0}>H_{x}\right) \geq \mathrm{P}\left(H_{x} \leq n \mid H_{0}>H_{x}\right) .
$$

Let us stress that this proposition is general, as it does not depend on the position of the obstacles.

Proof of Proposition 3.6. Let $x \in \mathbb{N}$. We first remark that the stopped process $\left(S_{k \wedge H_{x}}\right)_{k \in \mathbb{N}_{0}}$ is still a Markov chain under $\mathrm{P}^{\beta}\left(\cdot \mid \sigma \wedge H_{0}>H_{x}\right)$, with 0 as initial state, $x$ as absorbing state, and transition probabilities given by

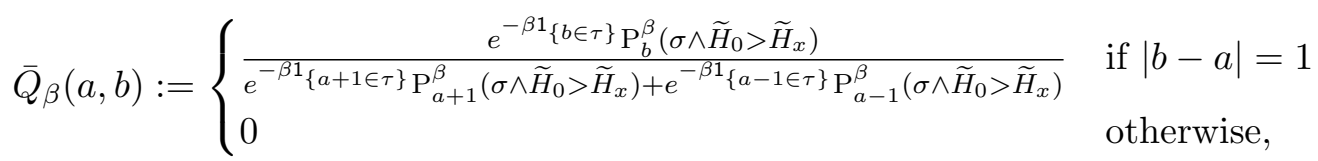


where $1 \leq a<x$ and $\widetilde{H}_{z}:=\inf \left\{n \in \mathbb{N}_{0}: S_{n}=z\right\}$. By applying the strong Markov property at $H_{a+1}$, observe that

(3.34)

$$
\frac{\bar{Q}_{\beta}(a, a-1)}{\bar{Q}_{\beta}(a, a+1)}=\frac{e^{-\beta 1_{\{a-1 \in \tau\}} \mathrm{P}_{a-1}^{\beta}\left(\sigma \wedge \widetilde{H}_{0}>\widetilde{H}_{x}\right)}}{e^{-\beta 1_{\{a+1 \in \tau\}} \mathrm{P}_{a+1}^{\beta}\left(\sigma \wedge \widetilde{H}_{0}>\widetilde{H}_{x}\right)}}=e^{-\beta 1_{\{a-1 \in \tau\}}} \mathrm{P}_{a-1}^{\beta}\left(\sigma \wedge \widetilde{H}_{0}>H_{a+1}\right),
$$

and note that this ratio is non-increasing in $\beta$, for all $1 \leq a<x$. We may deduce by a standard coupling argument that $H_{x}$ is stochastically smaller under $\mathrm{P}^{\beta}\left(\cdot \mid \sigma \wedge H_{0}>H_{x}\right)$ than under $\mathrm{P}\left(\cdot \mid H_{0}>H_{x}\right)$, which corresponds to the case $\beta=0$. This concludes the proof.

3.4. Two technical lemmas. Recall the notations in (3.10) and (3.11).

Lemma 3.7. Let $\left(\tau_{i}\right)_{i \in \mathbb{N}_{0}}$ be an increasing sequence of integers with $\tau_{0}=0$ and let $t_{i}=$ $\tau_{i}-\tau_{i-1}$ for all $i \in \mathbb{N}$. Define the function $f: z \in(0, \pi) \mapsto z / \sin (z)$. Let $n \in \mathbb{N}$. For $\varepsilon>0$ small enough, there exist $\alpha=\alpha(\varepsilon)>0, C>0$ and $\mathcal{T}_{0}(\varepsilon) \in \mathbb{N}$ such that for $t>\mathcal{T}_{0}(\varepsilon) \vee \max _{0 \leq i \leq n} t_{i}$ and $\phi=\frac{\pi^{2}}{2 t^{2}}$,

$$
\frac{Q_{i j}(\phi)}{Q_{i j}(0)} \leq \begin{cases}\exp (\varepsilon) & \text { if } i \neq j \text { and } t_{i j} \leq \alpha t, \text { or } i=j \\ 2 f\left(\pi \frac{\max _{0 \leq i \leq n} t_{i}}{t}\left(1+\frac{C}{t^{2}}\right)\right) & \text { else. }\end{cases}
$$

The ratio in (3.35) is the positive Laplace transform of the hitting time of $\tau$ for the walk conditioned to go from $\tau_{i}$ to $\tau_{j}$. As we will consider large values of $t$ and thus small values of $\phi$, Lemma 3.7 can be understood as a control of this transform near 0 .

Proof of Lemma 3.7. We consider $\varepsilon>0$ small enough (it will appear in the following how small it has to be). Let us start with the case $i \neq j$. From the explicit expressions of the Laplace transforms, see e.g. (A.5) in Caravenna and Pétrélis [5], we get

$$
Q_{i j}(\phi)=\frac{\tan \Delta}{2 \sin \left(t_{i j} \Delta\right)}, \quad \text { where } \Delta=\Delta(\phi)=\arctan \left(\sqrt{e^{2 \phi}-1}\right),
$$

and we note that

$$
Q_{i j}(0)=\frac{1}{2 t_{i j}} .
$$

Let us notice that (3.36) is well-defined if $t_{i j} \Delta<\pi$, which occurs as soon as $t$ is large enough. Indeed, by expanding $\Delta$ we see that there exists a constant $C>0$ and $\mathcal{T}_{1} \in \mathbb{N}$ such that for $t \geq \mathcal{T}_{1}$,

$$
\Delta \leq \frac{\pi}{t}\left(1+\frac{C}{t^{2}}\right)
$$

If we assume moreover that $t>t_{i j}$, we obtain, as $t$ and $t_{i j}$ are integers,

$$
\frac{t_{i j} \Delta}{\pi} \leq \frac{t-1}{t}\left(1+\frac{C}{t^{2}}\right)=1-\frac{1+o(1)}{t}<\frac{1}{1+\varepsilon},
$$

provided $t$ is larger than some $\mathcal{T}_{1}(\varepsilon) \in \mathbb{N}$. For the rest of the proof, we assume that $t>\mathcal{T}_{1} \vee \mathcal{T}_{1}(\varepsilon) \vee \max \left\{t_{i}: 0 \leq i \leq n\right\}$

By combining (3.36) and (3.37), we now obtain

$$
\frac{Q_{i j}(\phi)}{Q_{i j}(0)}=\frac{t_{i j} \tan (\Delta)}{\sin \left(t_{i j} \Delta\right)} .
$$

By using (3.38) and expanding tan to first order, there exists $\mathcal{T}_{2}(\varepsilon)$ such that for $t \geq \mathcal{T}_{2}(\varepsilon)$,

$$
\tan (\Delta) \leq(1+\varepsilon) \Delta .
$$


By adding this latter condition on $t$, we get, since $f$ is increasing,

$$
\begin{aligned}
\frac{Q_{i j}(\phi)}{Q_{i j}(0)} & \leq(1+\varepsilon) \frac{t_{i j} \Delta}{\sin \left(t_{i j} \Delta\right)} \\
& =(1+\varepsilon) f\left(t_{i j} \Delta\right) \\
& \leq(1+\varepsilon) f\left(\pi \frac{t_{i j}}{t}\left(1+\frac{C}{t^{2}}\right)\right) .
\end{aligned}
$$

As $\varepsilon<1$, that concludes the proof of the second inequality in our statement. To get the first inequality when $i \neq j$, notice first that, as $f(z) \rightarrow 1$ when $z \rightarrow 0$, there exists $z_{\varepsilon}$ such that $(1+\varepsilon) f(z) \leq \exp (\varepsilon)$ for $z \leq z_{\varepsilon}$. Therefore, it is enough to define

$$
\alpha(\varepsilon)=\frac{z_{\varepsilon}}{\pi(1+\varepsilon)},
$$

assume that $t>\mathcal{T}_{3}(\varepsilon):=(C / \varepsilon)^{1 / 2}$ and use (3.42) to get what we need.

We are left with the case $i=j$. Again, with the help of (A.5) in Caravenna and Pétrélis [5],

$$
Q_{i i}(\phi)=1-\frac{1}{2} \frac{\tan (\Delta)}{\tan \left(t_{i-1, i} \Delta\right)}-\frac{1}{2} \frac{\tan (\Delta)}{\tan \left(t_{i, i+1} \Delta\right)},
$$

where $\Delta$ is defined as in (3.36). We thereby retrieve the standard formula:

$$
Q_{i i}(0)=1-\frac{1}{2 t_{i-1, i}}-\frac{1}{2 t_{i, i+1}} .
$$

Note that it is enough to treat the case $t_{i, i+1}=t_{i-1, i}$ since

$$
Q_{i i}(\phi)=\frac{1}{2}\left(1-\frac{\tan (\Delta)}{\tan \left(t_{i-1, i} \Delta\right)}\right)+\frac{1}{2}\left(1-\frac{\tan (\Delta)}{\tan \left(t_{i, i+1} \Delta\right)}\right) .
$$

We may now consider the ratio

$$
\frac{Q_{i i}(\phi)}{Q_{i i}(0)}=\frac{1-\frac{\tan (\Delta)}{\tan \left(t_{i, i+1} \Delta\right)}}{1-\frac{1}{t_{i, i+1}}} .
$$

By choosing $t \geq \mathcal{T}_{2}(\varepsilon)$ and expanding tan to first order, we obtain

$$
1-\frac{\tan (\Delta)}{\tan \left(t_{i, i+1} \Delta\right)} \leq\left\{\begin{array}{lll}
1-\frac{\Delta}{\tan \left(t_{i, i+1} \Delta\right)} & \text { if } & t_{i, i+1} \Delta \leq \frac{\pi}{2}, \\
1-(1+\varepsilon) \frac{\Delta}{\tan \left(t_{i, i+1} \Delta\right)} & \text { if } & \frac{\pi}{2}<t_{i, i+1} \Delta<\pi .
\end{array}\right.
$$

We recall that our conditions on $t$ guarantee that $t_{i, i+1} \Delta<\pi$. The reason why we split cases above is that tan changes sign at the value $\pi$. We further make a dichotomy: (i) $t$ is large and $t_{i+1}$ is at least $\varepsilon t$ and (ii) $t$ is large and $t_{i+1}$ less than $\varepsilon t$. Let us start with (i). If actually $t_{i, i+1} \Delta<\pi / 2$, we may simply bound the denominator in (3.47) by 1 . Otherwise, we note that $z \mapsto-z / \tan (z)$ is increasing on $(\pi / 2, \pi)$, so we may write, as $t_{i, i+1} \Delta<\pi /(1+\varepsilon)$ by $(3.39)$,

$$
1-(1+\varepsilon) \frac{\Delta}{\tan \left(t_{i, i+1} \Delta\right)} \leq 1-\frac{1}{t_{i, i+1}} \frac{\pi}{\tan (\pi /(1+\varepsilon))} .
$$

Thus, if we define

$$
t_{4}(\varepsilon)=\frac{3}{\varepsilon^{2}}\left(\frac{\pi}{|\tan (\pi /(1+\varepsilon))|} \vee 1\right)
$$


and assume that $t>\mathcal{T}_{4}(\varepsilon)$, we obtain

$$
\frac{Q_{i i}(\phi)}{Q_{i i}(0)} \leq \frac{1-\frac{1}{t_{i, i+1}} \frac{\pi}{\tan (\pi /(1+\varepsilon))}}{1-\frac{1}{t_{i, i+1}}} \leq \frac{1+\varepsilon / 3}{1-\varepsilon / 3}=1+\frac{2}{3} \varepsilon+o(\varepsilon), \quad \varepsilon \rightarrow 0,
$$

which is smaller than $\exp (\varepsilon)$ when $\varepsilon$ is small enough. We now deal with (ii) and to this end we assume $t \geq \mathcal{T}_{2}(\varepsilon)$ and $t_{i, i+1} \leq \varepsilon t$, in which case we expand $\tan \left(t_{i, i+1} \Delta\right)$. By using (3.38) and assuming that $t>\mathcal{T}_{3}(\varepsilon)$ we get $t_{i, i+1} \Delta \leq \varepsilon(1+\varepsilon) \pi$. Thus, there exists a constant $C=C(\varepsilon)>0$ such that

$$
\tan \left(t_{i, i+1} \Delta\right) \leq t_{i, i+1} \Delta+C\left(t_{i, i+1} \Delta\right)^{3}, \quad \text { so } 1-\frac{\Delta}{\tan \left(t_{i, i+1} \Delta\right)} \leq 1-\frac{1}{t_{i, i+1}}\left(1-C t_{i, i+1}^{2} \Delta^{2}\right) .
$$

Finally, since $t_{i, i+1} \geq 2$ necessarily,

$$
\frac{Q_{i i}(\phi)}{Q_{i i}(0)} \leq 1+2 C t_{i, i+1} \Delta^{2} \leq 1+2 C \varepsilon(1+\varepsilon) \Delta .
$$

Now we pick $t \geq \mathcal{T}_{5}(\varepsilon)$ such that $\Delta \leq[2 C(1+\varepsilon)]^{-1}$ and we get the claim.

We conclude the proof by setting $\mathcal{T}_{0}(\varepsilon)=\max \left(\mathcal{T}_{1}, \mathcal{T}_{1}(\varepsilon), \mathcal{T}_{2}(\varepsilon), \mathcal{T}_{3}(\varepsilon), \mathcal{T}_{4}(\varepsilon), \mathcal{T}_{5}(\varepsilon)\right)$.

Recall the notations in (3.2) and (3.11). Given $\alpha>0$ and $k \in \mathbb{N}$, we define a set of bad edges as

$$
\mathcal{B}_{k, \alpha}=\left\{1 \leq x, y \leq i(k): x \neq y, t_{x, y}>\alpha t_{k}^{*}\right\}
$$

and its cardinal

$$
L_{k, \alpha}=\left|\mathcal{B}_{k, \alpha}\right| .
$$

These bad edges correspond to the second case in Lemma 3.7. Recall also (3.7) and (3.8). The following lemma controls the visits to the bad edges:

Lemma 3.8. Let $\left(\tau_{i}\right)_{i \in \mathbb{N}_{0}}$ be an increasing sequence of integers with $\tau_{0}=0$ and let $t_{i}=$ $\tau_{i}-\tau_{i-1}$ for all $i \in \mathbb{N}$. There exists a function $h$ such that, for any $A>0, k \geq 0$, and $\alpha>0$, if $t_{k}^{*}>h\left(A, L_{k, \alpha}, \alpha\right)$ then

$$
\mathrm{E}^{\beta}\left(A^{\sharp\left\{i \leq \zeta_{k}^{*}:\left\{X_{i-1}, X_{i}\right\} \in \mathcal{B}_{k, \alpha}\right\}} 1_{\left\{\zeta_{k}^{*}<\zeta_{0} \wedge \mathcal{N}\right\}}\right) \leq 2 A^{L_{k, \alpha}} \tau_{k}^{*} \mathrm{P}^{\beta}\left(\zeta_{k}^{*}<\zeta_{0} \wedge \mathcal{N}\right) .
$$

Proof of Lemma 3.8. We start with the case $L_{k, \alpha}=1$ and denote by $(s, s+1)$ the bad edge. By using the geometric nature of $\mathcal{N}$ and applying the Markov property at $\zeta_{s+1}$, we get

$$
\begin{gathered}
\mathrm{E}^{\beta}\left(A^{\sharp\left\{i \leq \zeta_{k}^{*}:\left\{X_{i-1}, X_{i}\right\} \in \mathcal{B}_{k, \alpha}\right\}} 1_{\left\{\zeta_{k}^{*}<\zeta_{0} \wedge \mathcal{N}\right\}}\right)=\mathrm{E}^{\beta}\left(A^{\sharp\left\{i \leq \zeta_{s+1}:\left\{X_{i-1}, X_{i}\right\}=\{s, s+1\}\right\}} 1_{\left\{\zeta_{k}^{*}<\zeta_{0} \wedge \mathcal{N}\right\}}\right) \\
\quad \leq \mathrm{P}^{\beta}\left(\zeta_{s+1}<\zeta_{0} \wedge \mathcal{N}\right) A \mathrm{E}_{\tau_{s+1}}^{\beta}\left(A^{\sharp\left\{i \leq \zeta_{s+1}:\left\{X_{i-1}, X_{i}\right\}=\{s, s+1\}\right\}} 1_{\left\{\zeta_{k}^{*}<\zeta_{0} \wedge \mathcal{N}\right\}}\right),
\end{gathered}
$$

and we now focus on the last factor in the line above. By considering the consecutive visits of $X$ to $s+1$, we may write

$$
\mathrm{E}_{\tau_{s+1}}^{\beta}\left(A^{\sharp\left\{i \leq \zeta_{s+1}:\left\{X_{i-1}, X_{i}\right\}=\{s, s+1\}\right\}} 1_{\left\{\zeta_{k}^{*}<\zeta_{0} \wedge \mathcal{N}\right\}}\right)=\mathrm{E}\left(v^{G}\right) \mathrm{P}_{\tau_{s+1}}\left(\zeta_{k}^{*}<\mathcal{N} \mid \zeta_{k}^{*}<\zeta_{s+1}\right),
$$

where $G$ is a $\mathbb{N}_{0}$-valued geometric random variable with parameter $\mathrm{P}_{\tau_{s+1}}\left(\zeta_{k}^{*}<\zeta_{s+1}\right)$ and

$$
v=\mathrm{E}_{\tau_{s+1}}\left(A^{\sharp\left\{i \leq \zeta_{s+1}:\left\{X_{i-1}, X_{i}\right\}=\{s, s+1\}\right\}} 1_{\left\{\zeta_{s+1}<\mathcal{N}\right\}} \mid \zeta_{s+1}<\zeta_{k}^{*}\right) .
$$


We are going to show now that $v \leq 1$ when $t_{k}^{*} \geq h_{0}(A, \alpha)$ where

$$
h_{0}(A, \alpha)=\frac{A^{2}}{2 \alpha e^{\beta}\left(e^{\beta}-1\right)} \text {. }
$$

To this end, note that

$$
v \leq \frac{1}{2} e^{-\beta}+\frac{1}{2}\left(1-\frac{1}{t_{s, s+1}}\right) e^{-\beta}+\frac{1}{2 t_{s, s+1}} A^{2} e^{-2 \beta} .
$$

Indeed, the first term is given by walks which make their first step to the right. The second term comes from those who make their first step to the left but come back to $\tau_{s+1}$ before hitting $\tau_{s}$, whereas the third term comes from the walks who hit $\tau_{s}$ before coming back to $\tau_{s+1}$. Then, as $t_{s, s+1} \geq \alpha t_{k}^{*}$,

$$
v \leq e^{-\beta}+\frac{A^{2} e^{-2 \beta}}{2 \alpha t_{k}^{*}},
$$

which, by (3.60), proves that $v \leq 1$. To complete the proof in this case, we write

$$
\begin{aligned}
\text { r.h.s }(3.57) & \leq A \mathrm{P}^{\beta}\left(\zeta_{s+1}<\zeta_{0} \wedge \mathcal{N}\right) \mathrm{P}_{\tau_{s+1}}\left(\zeta_{k}^{*}<\mathcal{N} \mid \zeta_{k}^{*}<\zeta_{s+1}\right) \\
& \leq A \mathrm{P}^{\beta}\left(\zeta_{s+1}<\zeta_{0} \wedge \mathcal{N}\right) \frac{\mathrm{P}_{\tau_{s+1}}^{\beta}\left(\zeta_{k}^{*}<\mathcal{N} \wedge \zeta_{s+1}\right)}{\mathrm{P}_{\tau_{s+1}}\left(\zeta_{k}^{*}<\zeta_{s+1}\right)} \\
& \leq 2 A\left(\tau_{k}^{*}-\tau_{s+1}\right) \mathrm{P}^{\beta}\left(\zeta_{s+1}<\zeta_{0} \wedge \mathcal{N}\right) \mathrm{P}_{\tau_{s+1}}^{\beta}\left(\zeta_{k}^{*}<\mathcal{N} \wedge \zeta_{s+1}\right) \\
& \leq 2 A \tau_{k}^{*} \mathrm{P}^{\beta}\left(\zeta_{k}^{*}<\zeta_{0} \wedge \mathcal{N}\right) .
\end{aligned}
$$

Let us now conclude the proof in the general case $L_{k, \alpha} \geq 1$. Our strategy is to decouple the contribution of each bad set by Holdër's inequality and reduce the problem to the case $L_{k, \alpha}=1$ with $A$ replaced by $A^{L}$. Indeed, if we note

$$
\mathcal{B}_{k, \alpha}=\left\{\left(s_{\ell}, s_{\ell+1}\right): 1 \leq \ell \leq L, 1 \leq s_{\ell}<i(k)\right\},
$$

and suppose that $t_{k}^{*} \geq h\left(A, L_{k, \alpha}, \alpha\right)$, where

$$
h(A, L, \alpha):=h_{0}\left(A^{L}, \alpha\right)=\frac{A^{2 L}}{2 \alpha e^{\beta}\left(e^{\beta}-1\right)},
$$

we get

$$
\begin{aligned}
& \mathrm{E}^{\beta}\left(A^{\sharp\left\{i \leq \zeta_{k}^{*}:\left\{X_{i-1}, X_{i}\right\} \in \mathcal{B}_{k, \alpha}\right\}} 1_{\left\{\zeta_{k}^{*}<\zeta_{0} \wedge \mathcal{N}\right\}}\right) \\
& \leq \prod_{i=1}^{L_{k, \alpha}} \mathrm{E}^{\beta}\left(\left(A^{L_{k, \alpha}}\right)^{\sharp\left\{i \leq \zeta_{k}^{*}:\left\{X_{i-1}, X_{i}\right\}=\left\{s_{\ell}, s_{\ell+1}\right\}\right\}} 1_{\left\{\zeta_{k}^{*}<\zeta_{0} \wedge \mathcal{N}\right\}}\right)^{1 / L_{k, \alpha}} \\
& \leq 2 A^{L_{k, \alpha}} \tau_{k}^{*} \mathrm{P}^{\beta}\left(\zeta_{k}^{*}<\zeta_{0} \wedge \mathcal{N}\right) .
\end{aligned}
$$

This concludes the proof.

3.5. Good environments. We define here a notion of good environments, that are environments where it is possible to give a good control on the survival probability. We will show in Section 6.1 that these environments are typical, meaning that by tuning some parameters and considering $n$ large enough, their probability of occurence can be made arbitrarily close to one. 
Additional notations. Beforehand, recall the functions $f$ and $h$ introduced in Lemma 3.7 and Lemma 3.8. We define

$$
f_{k}:=2 f\left(\pi \frac{T_{k-1}^{*}}{T_{k}^{*}}\left[1+\frac{C}{\left(T_{k}^{*}\right)^{2}}\right]\right)
$$

that appears in the events $A_{n}^{(6)}$ and $A_{n}^{(7)}$ below. The constant $C$ above is the same as the one in (3.35) in Lemma 3.7. From (3.14) and (3.16), there exists (for a fixed $\beta$ ) a constant $C_{1}>0$ so that

$$
1 /\left(C_{1} t^{2}\right) \leq g(t) \wedge \phi(\beta, t) \leq g(t) \vee \phi(\beta, t) \leq C_{1} / t^{2}, \quad t \geq 1 .
$$

This constant appears in the event $A_{n}^{(9)}$. Finally, we define the exponent

$$
\kappa= \begin{cases}\frac{\gamma}{4} & \text { if } \gamma \leq 1 \\ \frac{1}{2 \gamma}-\frac{1}{4} & \text { if } 1<\gamma<2 \\ \frac{1}{2 \gamma} & \text { if } \gamma \geq 2,\end{cases}
$$

which appears in the event $A_{n}^{(1)}$.

Definition. Let $\delta, \varepsilon_{0}, \varepsilon, \eta>0$. The set of good environments, denoted by $\Omega_{n}\left(\delta, \varepsilon_{0}, \varepsilon, \eta\right)$, is defined as the intersection of the events defined below (we stress that $\alpha(\varepsilon)$ and $\mathcal{T}_{0}(\varepsilon)$ appearing here are the same as in Lemma 3.7 and $\mathcal{T}_{0}$ comes from Proposition 3.1):

$$
\Omega_{n}\left(\delta, \varepsilon_{0}, \varepsilon, \eta\right)=\bigcap_{i=1}^{11} A_{n}^{(i)}\left(\delta, \varepsilon_{0}, \varepsilon, \eta\right)
$$

with

$$
\begin{aligned}
A_{n}^{(1)} & = \begin{cases}\left\{\tau_{N^{1+\kappa}}^{2}<n^{1-\frac{\gamma \wedge(2-\gamma)}{4(\gamma+2)}}\right\} & \text { if } \gamma<2 \\
\left\{\tau_{N^{1+\kappa}}^{2}<n^{1+\frac{2 \gamma-1}{2(\gamma+2)}}\right\} & \text { if } \gamma \geq 2,\end{cases} \\
A_{n}^{(2)}\left(\varepsilon_{0}\right) & :=\left\{T_{k} \leq \varepsilon_{0}^{\frac{1}{2 \gamma}} N^{\frac{1}{\gamma}}, \quad \forall k \leq \varepsilon_{0} N\right\} \\
A_{n}^{(3)}\left(\varepsilon_{0}\right) & :=\left\{\tau_{N / \varepsilon_{0}}<n\right\} \\
A_{n}^{(4)}(\delta) & :=\left\{\exists \ell \in\{N, \ldots, 2 N\}: T_{\ell} \geq \mathcal{T}_{0} \vee \delta N^{\frac{1}{\gamma}}\right\} \\
A_{n}^{(5)}\left(\varepsilon_{0}, \varepsilon\right) & :=\left\{\forall k \in R_{\varepsilon_{0}}(n), T_{k}^{*}>\mathcal{T}_{0}(\varepsilon) \vee \varepsilon_{0}^{\frac{3}{2 \gamma}} N^{\frac{1}{\gamma}}\right\} \\
A_{n}^{(6)}\left(\varepsilon_{0}, \varepsilon\right) & :=\left\{\forall k \in R_{\varepsilon_{0}}(n), f_{k}^{L_{k}} \leq \exp \left(n^{\frac{\gamma}{2(\gamma+2)}}\right)\right\} \\
A_{n}^{(7)}\left(\varepsilon_{0}, \varepsilon\right) & :=\left\{\forall k \in R_{\varepsilon_{0}}(n), T_{k}^{*}>h\left(f_{k}, L_{k, \alpha(\varepsilon)}, \alpha(\varepsilon)\right)\right\} \\
A_{n}^{(8)}\left(\varepsilon_{0}\right) & :=\left\{\left|R\left(1, N / \varepsilon_{0}\right)\right| \leq\left[\log \left(N / \varepsilon_{0}\right)\right]^{2}\right\} \\
A_{n}^{(9)}(\delta) & :=\left\{|\lambda(2 N, \beta)-\lambda(\beta)| \leq \frac{C_{1}}{2 \delta^{2}}\right\} \\
A_{n}^{(10)}\left(\varepsilon_{0}, \varepsilon, \eta\right) & :=\left\{|\lambda(\ell-1, b)-\lambda(b)| \leq \frac{\varepsilon_{0} \eta}{2}, \forall \ell \geq \varepsilon_{0} N, b \in\{\beta, \beta-\varepsilon\}\right\} \\
A_{n}^{(11)}\left(\varepsilon_{0}\right) & :=\left\{\Pi_{N}\left(\left\{y>1 / \varepsilon_{0}\right\}\right)=0\right\} .
\end{aligned}
$$

We might omit some or all parameters of $\Omega_{n}$ when no confusion is possible to alight notations. Event $A_{n}^{(1)}$ and $A_{n}^{(4)}(\delta)$ are used to provide a lower bound on $Z_{n}$, see Proposition 4.1. Events from $A_{n}^{(2)}$ to $A_{n}^{(9)}$ are used to establish the upper bound, see Proposition 5.1. 
Finally, the events $A_{n}^{(10)}$ and $A_{n}^{(11)}$ are used in the conclusion of the proof, see (6.58) and (6.56) in Section 6.2.

\section{LOWER BOUND}

In this section we prove a lower bound on $Z_{n}$ that is an upper bound on $F_{n}$. Let us set for $\beta \geq 0, n \in \mathbb{N}$ and $\ell>1$,

$$
G_{n}^{\beta}(\ell)=-\frac{1}{N} \log \mathrm{P}^{\beta}\left(\sigma \wedge H_{0}>H_{\tau_{\ell-1}}\right)+g\left(T_{\ell}\right) \frac{n}{N}=\lambda(\ell-1, \beta) \frac{\ell-1}{N}+g\left(T_{\ell}\right) \frac{n}{N},
$$

where $\lambda(\ell, \beta), N$ and $g(\cdot)$ have been respectively defined in (2.1), (2.4) and (3.14). Recall the definition of $\kappa$ in (3.69). Our goal in this section is to prove

Proposition 4.1. On $\Omega_{n}(\delta)$,

$$
F_{n} \leq \min _{1<\ell \leq N^{1+\kappa}} G_{n}^{\beta}(\ell)+o(1)
$$

where o(1) denotes a deterministic function that goes to 0 when $n$ goes to $\infty$.

Actually, only $A_{n}^{(1)}$ and $A_{n}^{(4)}(\delta)$ are necessary in Proposition 4.1. In order to prove this proposition we need the following lemma, that states that the exponential cost of reaching level $N^{1+\kappa}$ (and so any level $\ell \leq N^{1+\kappa}$ ) before time $n$ is negligible compared to $N$.

Lemma 4.2. There exists a function $k: \mathbb{N} \mapsto(0, \infty)$ satisfying $k(x)=o(x)$ as $x \rightarrow \infty$ and for all $1<\ell<N^{1+\kappa}$ and $n \in \mathbb{N}$, on $\Omega_{n}$,

$$
\mathrm{P}\left(H_{\tau_{\ell}} \leq n \mid H_{\tau_{\ell}}<H_{0}\right) \geq \exp (-k(N)) .
$$

Proof of Lemma 4.2. Observe that

$$
\begin{aligned}
\mathrm{P}\left(H_{\tau_{\ell}} \leq n \mid H_{\tau_{\ell}}<H_{0}\right) & \geq \mathrm{P}\left(S_{n} \geq \tau_{\ell}, S_{k}>0,0<k \leq n\right) \\
& \geq \mathrm{P}\left(S_{n} \geq \tau_{\ell}\right) \mathrm{P}\left(S_{k}>0,0<k \leq n\right) \\
& \geq \mathrm{P}\left(S_{n} \geq \tau_{N^{1+\kappa}}\right) \mathrm{P}\left(S_{k}>0,0<k \leq n\right) .
\end{aligned}
$$

To go from the first to the second line we use the FKG inequality, since both events are non-decreasing coordinate-wise in the family of i.i.d. increments $\left(S_{i}-S_{i-1}\right)_{1 \leq i \leq n}$. As there exists some constant $C>0$ such that $\mathrm{P}\left(S_{k}>0,0<k \leq n\right) \geq C / \sqrt{n}$ we only focus on $\mathrm{P}\left(S_{n} \geq \tau_{N^{1+\kappa}}\right)$.

Assume first that $\gamma<2$. Then, we notice that, on $A_{n}^{(1)}, \tau_{N^{1+\kappa}}=o(\sqrt{n})$ when $n$ goes to infinity so that

$$
\mathrm{P}\left(S_{n} \geq \tau_{N^{1+\kappa}}\right)=\mathrm{P}\left(S_{n} \geq 0\right)-\mathrm{P}\left(0 \leq S_{n}<\tau_{N^{1+\kappa}}\right)=1 / 2+o(1) .
$$

Assume now that $\gamma \geq 2$. In this case, $\tau_{N^{1+\kappa}}$ is not anymore negligible compared to $\sqrt{n}$. However, on $A_{n}^{(1)}$, a standard large deviation estimate for the simple random walk yields

$$
\mathrm{P}\left(S_{n} \geq \tau_{N^{1+\kappa}}\right) \geq \exp \left(-C n^{\frac{2 \gamma-1}{2(\gamma+2)}}\right) .
$$

We may conclude by setting $k(N)=C N^{1-\frac{1}{2 \gamma}}$.

Proof of Proposition 4.1. We provide a lower bound on $Z_{n}$ by computing the cost of various strategies. Here is an informal description of the tested strategies: for $1<\ell \leq N^{1+\kappa}$, the walk reaches $\tau_{\ell-1}$ before time $n$ and before its death, which has a probability of order $e^{-\lambda \ell}$. Then, it remains confined in the gap $\left(\tau_{\ell-1}, \tau_{\ell}\right)$ until time $n$, with a probability of order $e^{-g\left(T_{\ell}\right) n}$. We finally optimise over $1<\ell \leq N^{1+\kappa}$. 
We may thus write for all $\ell>1$,

$$
Z_{n} \geq Z_{n}^{[\ell]}:=\mathrm{P}\left(H_{\tau_{\ell}} \wedge \sigma \wedge H_{0}>n \geq H_{\tau_{\ell-1}}\right),
$$

and then optimise over $1<\ell \leq N^{1+\kappa}$. Recall the definition of $\mathcal{T}_{0}$ in Proposition 3.1. By decomposing on the value of $H_{\tau_{\ell-1}}$, we have on $\Omega_{n}(\delta)$, for $n$ large enough and $1 \leq \ell \leq N^{1+\kappa}$ such that $T_{\ell} \geq \mathcal{T}_{0}$,

$$
\begin{aligned}
Z_{n}^{[\ell]} & \geq \sum_{0 \leq k \leq n} \mathrm{P}\left(\sigma \wedge H_{0}>H_{\tau_{\ell-1}}=k\right) \mathrm{P}_{\tau_{\ell-1}}\left(H_{\tau_{\ell}} \wedge H_{\tau_{\ell-1}}>n-k\right) \\
& \geq \sum_{0 \leq k \leq n} \mathrm{P}\left(\sigma \wedge H_{0}>H_{\tau_{\ell-1}}=k\right) \mathrm{P}_{\tau_{\ell-1}}\left(H_{\tau_{\ell}} \wedge H_{\tau_{\ell-1}}>n\right) \\
& \geq \mathrm{P}\left(\sigma \wedge H_{0}>H_{\tau_{\ell-1}}, H_{\tau_{\ell-1}} \leq n\right) \times \frac{c_{1}}{2 \sqrt{n}} e^{-g\left(T_{\ell}\right) n} \\
& \geq \mathrm{P}\left(\sigma \wedge H_{0}>H_{\tau_{\ell-1}}\right) \mathrm{P}\left(H_{\tau_{\ell-1}} \leq n \mid H_{0}>H_{\tau_{l-1}}\right) \times \frac{c_{1}}{2 \sqrt{n}} e^{-g\left(T_{\ell}\right) n} \\
& \geq \frac{c_{1}}{2 \sqrt{n}} \mathrm{P}\left(\sigma \wedge H_{0}>H_{\tau_{\ell-1}}\right) e^{-g\left(T_{\ell}\right) n-k(N)} .
\end{aligned}
$$

Note that we have used Proposition 3.1 to go from the second to the third line and Proposition 3.6 to go from the third to the fourth line. Finally, to go from the fourth to the fifth line we use Lemma 4.2 and the fact that the environment is in $\Omega_{n}(\delta)$. Therefore,

$$
F_{n} \leq-\frac{1}{N} \log \left(\frac{c_{1}}{2 \sqrt{n}}\right)+\frac{k(N)}{N}+\inf _{\substack{1<\ell \leq N^{1+\kappa} \\ T_{\ell} \geq \mathcal{T}_{0}}} G_{n}^{\beta}(\ell),
$$

where $G_{n}^{\beta}$ is defined in (4.1). From this definition, we observe that $G_{n}^{\beta}$ is the sum of two terms: one is increasing with $\ell$ and the other one is decreasing with $T_{\ell}$. Therefore the infimum above is achieved by some $\ell$ corresponding to a record value of $\left(T_{\ell}\right)_{\ell>1}$. Thus we may restrict the infimum in (4.9) to the $\ell$ 's in $\mathcal{R}\left(1, N^{1+\kappa}\right)$, which includes $\mathcal{R}(N, 2 N)$ for $n$ large enough. In turn, on $A_{n}^{(4)}(\delta)$, we may remove the condition $T_{\ell} \geq \mathcal{T}_{0}$ in the infimum. Finally, since

$$
-\frac{1}{N} \log \left(\frac{c_{1}}{2 \sqrt{n}}\right)+\frac{k(N)}{N}=o(1)
$$

as $N \rightarrow \infty$, the proof is complete.

\section{UPPER BOUND}

In this section we prove an upper bound on $Z_{n}$ or, equivalently, a lower bound on $F_{n}$. Recall the definitions in (3.4).

Proposition 5.1. Let $\varepsilon, \delta>0$. There exists $\varepsilon_{0}>0$ such that, on $\Omega_{n}\left(\delta, \varepsilon_{0}, \varepsilon\right)$,

$$
F_{n} \geq \min _{\ell \in \mathcal{R}_{\varepsilon_{0}}(n)} G_{n}^{\beta-\varepsilon}(\ell)+o(1) .
$$

where o(1) denotes a deterministic function that goes to 0 when $n$ goes to $\infty$ and $G_{n}^{\beta}$ is defined in (4.1). 
Before starting the proof of this proposition we need additional notations. Recall (3.1). We define the hitting time of the $k$-th record

$$
H_{k}^{*}=H_{\tau_{i(k)}}, \quad k \in \mathbb{N}_{0} .
$$

For all $n \geq 1$ and $k \geq 1$, we define

$$
Z_{n}^{(k)}=Z_{n}\left(H_{k}^{*} \leq n<H_{k+1}^{*}\right)
$$

where $Z_{n}(A)=\mathrm{P}^{\beta}\left(\sigma \wedge H_{\mathbb{Z}^{-}}>n, A\right)$ for any event $A$. The quantity above corresponds to the survival probability restricted to the event that the walker has reached the $k$-th record but not the $(k+1)$-th one. These events are clearly incompatible for different values of $k$.

Proof of Proposition 5.1. Let $\varepsilon, \delta>0$. We choose $\varepsilon_{0}$ small enough so that

$$
\begin{gathered}
\frac{\beta}{\varepsilon_{0}}>2\left(C_{1} \delta^{-2}+\lambda(\beta)\right), \\
\varepsilon_{0}^{-1 / \gamma}>4 C_{1}\left(C_{1} \delta^{-2}+\lambda(\beta)\right),
\end{gathered}
$$

(these technical conditions will become clear soon).

We have to prove that (5.1) is satisfied on $\Omega_{n}$. We thus consider until the end of this proof an environment that is in $\Omega_{n}$.

Step 1 . We first prove that we can actually consider only the records in $R_{\varepsilon_{0}}(n)$, that are the ones lying in the window $\left\{\varepsilon_{0} N, \cdots, N / \varepsilon_{0}\right\}$, see (5.9) for a precise formulation. We obtain, for $n$ large enough, a rough lower bound on $Z_{n}$ (recall (4.7)):

$$
\begin{aligned}
& Z_{n} \geq \max _{N \leq \ell \leq 2 N} Z_{n}^{[\ell]} \\
& \geq \max _{\substack{N \leq \ell \leq 2 N \\
T_{\ell} \geq \mathcal{T}_{0}}} \frac{c_{1}}{2 \sqrt{n}} \mathrm{P}\left(\sigma \wedge H_{0}>H_{\tau_{\ell-1}}\right) e^{-g\left(T_{\ell}\right) n-k(N)} \\
& \geq \frac{c_{1}}{2 \sqrt{n}} \mathrm{P}\left(\sigma \wedge H_{0}>H_{\tau_{2 N}}\right) e^{-k(N)} \max _{\substack{N<\ell<2 N \\
\bar{T}_{\ell} \geq T_{0}}} e^{-g\left(T_{\ell}\right) n} \\
& \geq \frac{c_{1}}{2 \sqrt{n}} \exp \left(-\left\{2 N \lambda(2 N, \beta)+k(N)+C_{1} \delta^{-2} N\right\}\right) .
\end{aligned}
$$

To go from the first to the second line we use (4.8), which is valid as the environment is in $A_{n}^{(1)} \cap A_{n}^{(4)}(\delta)$. To go from the third one to the last one we use the upper bound in (3.68) together with the assumption that the environment is in $A_{n}^{(4)}(\delta)$.

As the environment is in $A_{n}^{(9)}(\delta)$, we finally get

$$
Z_{n} \geq \frac{c_{1}}{2 \sqrt{n}} \exp \left\{-N\left(2 C_{1} \delta^{-2}+2 \lambda(\beta)+o(1)\right)\right\},
$$

where the $o(1)$ holds as $n \rightarrow \infty$. Observe that

$$
\sum_{k \in R\left(N / \varepsilon_{0}, \infty\right)} Z_{n}^{(k)} \leq e^{-\beta N / \varepsilon_{0}},
$$


while due to Proposition 3.4 and the fact that the environment is in $A_{n}^{(2)}\left(\varepsilon_{0}\right)$, we have for $n$ large enough,

$$
\begin{aligned}
\sum_{k \in R\left(0, \varepsilon_{0} N\right)} Z_{n}^{(k)} \leq Z_{n}\left(H_{\tau_{\varepsilon_{0} N}}>n\right) & \leq C n^{2}\left(\varepsilon_{0} N\right) \exp \left(-\phi\left(\beta, \max \left\{T_{i}, i \leq \varepsilon_{0} N\right\}\right) n\right) \\
& \leq C n^{2}\left(\varepsilon_{0} N\right) \exp \left(-\frac{n}{C_{1}\left(\varepsilon_{0}^{1 / 2 \gamma} N^{1 / \gamma}\right)^{2}}\right) \\
& \leq \exp \left(-\frac{\varepsilon_{0}^{-1 / \gamma} N}{2 C_{1}}\right)
\end{aligned}
$$

Combining (5.6), (5.7) and (5.8) and due to the choice of $\varepsilon_{0}$ in (5.4), we easily get that for $n$ large enough

$$
Z_{n} \leq 2 \sum_{k \in R_{\varepsilon_{0}}(n)} Z_{n}^{(k)}
$$

Step 2. The main work is thus to derive an upper bound on $Z_{n}^{(k)}$ for $k \in R_{\varepsilon_{0}}(n)$ (see (5.20) for a precise formulation).

Using the Markov property at time $H_{k}^{*}$ we observe that for $k \in R_{\varepsilon_{0}}(n)$

$$
\begin{aligned}
Z_{n}^{(k)} & =\mathrm{P}^{\beta}\left(\sigma \wedge H_{\mathbb{Z}^{-}}>n, H_{k}^{*} \leq n<H_{k+1}^{*}\right) \\
& =\sum_{m=0}^{n} \mathrm{P}^{\beta}\left(\sigma \wedge H_{\mathbb{Z}^{-}}>m, H_{k}^{*}=m\right) \mathrm{P}_{\tau_{i(k)}}^{\beta}\left(\sigma \wedge H_{k+1}^{*} \wedge H_{\mathbb{Z}^{-}}>n-m\right) .
\end{aligned}
$$

For all $m \geq 0$, by Proposition 3.4, we have on $A_{n}^{(3)}\left(\varepsilon_{0}\right)$, for $n$ large enough,

$$
\begin{aligned}
\mathrm{P}_{\tau_{i(k)}}^{\beta}\left(\sigma \wedge H_{k+1}^{*} \wedge H_{\mathbb{Z}^{-}}>n-m\right) & \leq C(n-m)^{2} i(k+1) e^{-\phi\left(\beta ; \max \left\{T_{i}: 0 \leq i<i(k+1)\right\}\right)(n-m)} \\
& \leq C n^{3} e^{-\phi\left(\beta, T_{k}^{*}\right)(n-m)} .
\end{aligned}
$$

It remains to bound $\mathrm{P}^{\beta}\left(\sigma \wedge H_{\mathbb{Z}^{-}}>m, H_{k}^{*}=m\right)$ for $0 \leq m \leq n$. Recall (3.7) and (3.8). By using Tchebychev's inequality in the first line and then conditioning on $X$, we obtain for $\phi:=\frac{\pi^{2}}{2\left(T_{k}^{*}\right)^{2}}$,

$$
\begin{aligned}
& \mathrm{P}^{\beta}\left(\sigma \wedge H_{\mathbb{Z}^{-}}>m, H_{k}^{*}=m\right)=\mathrm{P}^{\beta}\left(\zeta_{k}^{*}<\zeta_{0} \wedge \mathcal{N}, H_{k}^{*}=m\right) \\
& \leq e^{-\phi m} \mathrm{E}^{\beta}\left(e^{\phi H_{k}^{*}} 1_{\left\{\zeta_{k}^{*}<\zeta_{0} \wedge \mathcal{N}\right\}}\right) \\
& \leq e^{-\phi m} \mathrm{E}^{\beta}\left(\mathrm{E}\left(e^{\phi H_{k}^{*}} \mid X\right) 1_{\left\{\zeta_{k}^{*}<\zeta_{0} \wedge \mathcal{N}\right\}}\right) \\
& \leq e^{-\phi m} \mathrm{E}^{\beta}\left(\prod_{1 \leq i \leq \zeta_{k}^{*}} \mathrm{E}\left(e^{\phi(\Delta \theta)_{i}} \mid X_{i-1}, X_{i}\right) 1_{\left\{\zeta_{k}^{*}<\zeta_{0} \wedge \mathcal{N}\right\}}\right),
\end{aligned}
$$

where $(\Delta \theta)_{i}=\theta_{i}-\theta_{i-1}$ for $i \geq 1$. Next, by integrating out $\mathcal{N}$ we obtain

$$
\mathrm{E}^{\beta}\left(\prod_{1 \leq i \leq \zeta_{k}^{*}} \mathrm{E}\left(e^{\phi(\Delta \theta)_{i}} \mid X_{i-1}, X_{i}\right) 1_{\left\{\zeta_{k}^{*}<\zeta_{0} \wedge \mathcal{N}\right\}}\right)=\mathrm{E}\left(\prod_{1 \leq i \leq \zeta_{k}^{*}} e^{-\beta} \frac{Q_{X_{i-1}, X_{i}}(\phi)}{Q_{X_{i-1}, X_{i}}(0)} 1_{\left\{\zeta_{k}^{*}<\zeta_{0}\right\}}\right)
$$

with notations similar to (3.10). 
On $A_{n}^{(5)}\left(\varepsilon_{0}, \varepsilon\right)$ the assumptions of Lemma 3.7 are valid (with $T_{k}^{*}$ playing the role of $t$ ), which provides $\alpha>0$. Recall the definition of $\mathcal{B}_{k, \alpha}$ in (3.54). We obtain

$$
\begin{aligned}
& \mathrm{E}\left(\prod_{1 \leq i \leq \zeta_{k}^{*}} e^{-\beta} \frac{Q_{X_{i-1}, X_{i}}(\phi)}{Q_{X_{i-1}, X_{i}}(0)} 1_{\left\{\zeta_{k}^{*}<\zeta_{0}\right\}}\right) \\
& \quad \leq \mathrm{E}^{\beta-\varepsilon}\left(2 f\left(\pi \frac{T_{k-1}^{*}}{T_{k}^{*}}\left(1+\frac{C}{\left(T_{k}^{*}\right)^{2}}\right)\right)^{\sharp\left\{i \leq \zeta_{k}^{*}:\left\{X_{i-1}, X_{i}\right\} \in \mathcal{B}_{k, \alpha}\right\}} 1_{\left\{\zeta_{k}^{*}<\zeta_{0} \wedge \mathcal{N}\right\}}\right) .
\end{aligned}
$$

Recall the definition of $f_{k}$ in (3.67). On $A_{n}^{(7)}\left(\varepsilon_{0}, \varepsilon\right)$ the assumptions of Lemma 3.8 (with $f_{k}$ playing the role of $A$ ) are satisfied and from (5.14) we obtain that on $A_{n}^{(3)}\left(\varepsilon_{0}\right)$

$$
\begin{aligned}
\mathrm{E}\left(\prod_{1 \leq i \leq \zeta_{k}^{*}} e^{-\beta} \frac{Q_{X_{i-1}, X_{i}}(\phi)}{Q_{X_{i-1}, X_{i}}(0)} 1_{\left\{\zeta_{k}^{*}<\zeta_{0}\right\}}\right) & \leq \mathrm{E}^{\beta-\varepsilon}\left(f_{k}^{\sharp\left\{i \leq \zeta_{k}^{*}:\left\{X_{i-1}, X_{i}\right\} \in \mathcal{B}_{k, \alpha}\right\}} 1_{\left\{\zeta_{k}^{*}<\zeta_{0} \wedge \mathcal{N}\right\}}\right) \\
& \leq 2 f_{k}^{L, \alpha} n \mathrm{P}^{\beta-\varepsilon}\left(\zeta_{k}^{*}<\zeta_{0} \wedge \mathcal{N}\right) .
\end{aligned}
$$

Finally, combining (5.10), (5.11), (5.12) and (5.15) we obtain for $k \in R_{\varepsilon_{0}}(n)$

$$
\begin{aligned}
Z_{n}^{(k)} & \leq 2 C n^{4} f_{k}^{L_{k, \alpha}} e^{-\phi\left(\beta, T_{k}^{*}\right) n} \mathrm{P}^{\beta-\varepsilon}\left(\zeta_{k}^{*}<\zeta_{0} \wedge \mathcal{N}\right) \sum_{m=0}^{n} e^{-\left(\frac{\pi^{2}}{2\left(T_{k}^{*}\right)^{2}}-\phi\left(\beta, T_{k}^{*}\right)\right) m} \\
& \leq 2 C n^{4} f_{k}^{L_{k, \alpha}} e^{-G_{n}^{\beta-\varepsilon}(i(k)) N} e^{-\left(\phi\left(\beta ; T_{k}^{*}\right)-g\left(T_{k}^{*}\right)\right) n} \sum_{m=0}^{n} e^{-\left(\frac{\pi^{2}}{2\left(T_{k}^{*}\right)^{2}}-\phi\left(\beta, T_{k}^{*}\right)\right) m} .
\end{aligned}
$$

On $A_{n}^{(5)}\left(\varepsilon_{0}, \varepsilon\right)$ we control both the errors $\phi\left(\beta ; T_{k}^{*}\right)-g\left(T_{k}^{*}\right)$ and $\phi\left(\beta, T_{k}^{*}\right)-\frac{\pi^{2}}{2\left(T_{k}^{*}\right)^{2}}$. Indeed due to (3.14) and (3.16) there exists some constant $C(\beta)$ such that for $t$ large enough (depending on $\beta$ ),

$$
\left|\phi(\beta ; t)-\frac{\pi^{2}}{2 t^{2}}\right| \vee|\phi(\beta ; t)-g(t)|<\frac{C(\beta)}{t^{3}},
$$

and we obtain that on $A_{n}^{(5)}\left(\varepsilon_{0}, \varepsilon\right)$ and for $n$ large enough

$$
\left|\phi\left(\beta ; T_{k}^{*}\right)-\frac{\pi^{2}}{2\left(T_{k}^{*}\right)^{2}}\right| \vee\left|\phi\left(\beta ; T_{k}^{*}\right)-g\left(T_{k}^{*}\right)\right| \leq \frac{C(\beta)}{\varepsilon_{0}^{9 / 2 \gamma} N^{3 / \gamma}} .
$$

From (5.16) we thus obtain for $n$ large enough and $k \in R_{\varepsilon_{0}}(n)$

$$
Z_{n}^{(k)} \leq 2 C n^{5} f_{k}^{L, \alpha} \exp \left\{-N \min _{\ell \in \mathcal{R}_{\varepsilon_{0}}(n)} G_{n}^{\beta-\varepsilon}(\ell)+C(\beta) \varepsilon_{0}^{-9 / 2 \gamma} n^{\frac{\gamma-1}{\gamma+2}}\right\} .
$$

We also recall that on $A_{n}^{(6)}\left(\varepsilon_{0}, \varepsilon\right), f_{k}^{L_{k, \alpha}} \leq e^{n^{\frac{\gamma}{2(\gamma+2)}}}$ so that finally for $k \in R_{\varepsilon_{0}}(n)$, and for $n$ large enough,

$$
Z_{n}^{(k)} \leq 2 C n^{5} \exp \left(-N \min _{\ell \in \mathcal{R}_{\varepsilon_{0}}(n)} G_{n}^{\beta-\varepsilon}(\ell)+o(N)\right) .
$$

Step 3. It remains to sum this last equation for $k \in R_{\varepsilon_{0}}(n)$. As the environment is in $A_{n}^{(8)}\left(\varepsilon_{0}\right)$, we finally obtain that for $n$ large enough

$$
Z_{n} \leq \varepsilon_{0}^{-1} 2 C n^{6} \exp \left(-N \min _{\ell \in \mathcal{R}_{\varepsilon_{0}}(n)} G_{n}^{\beta-\varepsilon}(\ell)+o(N)\right)
$$


and

$$
F_{n} \geq \min _{\ell \in \mathcal{R}_{\varepsilon_{0}}(n)} G_{n}^{\beta-\varepsilon}(\ell)+o(1),
$$

where the $o(1)$ holds as $n \rightarrow \infty$.

\section{Proof of Theorem 2.2}

This section is divided in two parts. In the first one we establish that, for $n$ large enough, environments are good with probability arbitrary close from 1 . The second one is devoted to the proof our main result Theorem 2.2. Due to the control on the environment we can actually restrict our analysis to the event of good environments so that results of Proposition 4.1 and 5.1 are in force and provide a precise control on $Z_{n}$.

6.1. Environments are good with high probability. The aim of this part is to prove the following proposition that assures a control of the probability that an environment is good:

Proposition 6.1. For all $\theta>0$ there exists $\delta$ and $\varepsilon_{1}(\delta)$ small enough such that for all $\varepsilon_{0}<\varepsilon_{1}(\delta)$, for all $\varepsilon, \eta>0$

$$
\liminf _{n \rightarrow \infty} \mathbb{P}\left(\Omega_{n}\left(\delta, \varepsilon_{0}, \varepsilon, \eta\right)\right) \geq 1-\theta .
$$

We first establish various results on the records of the sequence $\left(T_{i}\right)_{i \geq 1}$.

Preliminaries on records. We say that $n \in \mathbb{N}$ is a record if $n=1$ or $n \geq 2$ and $T_{n}>$ $\max \left\{T_{1}, \ldots, T_{n-1}\right\}$. Let us define

$$
I_{n}= \begin{cases}1 & \text { if } n \text { is a record } \\ 0 & \text { otherwise }\end{cases}
$$

the indicator of a record, and

$$
\mathfrak{R}_{n}=\sum_{k=1}^{n} I_{k}=|R(1, n)|
$$

the number of records between 1 and $n$. It was noticed (see Rényi [15]) that when $T_{1}$ is a continuous random variable, the $I_{n}$ 's are independent Bernoulli random variables with mean $\mathrm{E}\left(I_{n}\right)=1 / n$. However, we deal here with the discrete case, where this simple structure breaks down because of the possibility of ties. Actually, this case seems to have attracted relatively little attention (see however [12] and references therein). In this section, we provide some results in the discrete case (moments and deviations from the mean for the number of records) that will be useful later, though we do not aim for optimality. We start with:

Proposition 6.2. For all $p \in \mathbb{N}$ and $1 \leq n_{1}<n_{2}<\ldots<n_{p}$,

$$
\mathrm{E}\left(I_{n_{1}} \ldots I_{n_{p}}\right) \leq\left(1 / n_{1}\right) \ldots\left(1 / n_{p}\right) .
$$

As a consequence we obtain:

Proposition 6.3. For $b>1$ there exists a positive constant $c=c(b)$ (which we may choose smaller but arbitrarily close to $\left.\sup _{\lambda>0}\left\{b \lambda+1-e^{\lambda}\right\}=1+b(\ln b-1)>0\right)$ such that for $n$ large enough,

$$
\mathrm{P}\left(\Re_{n} \geq b \log n\right) \leq n^{-c} .
$$


Proof of Proposition 6.2. We prove it by iteration on $p \geq 1$ and thus start with the case $p=1$. Let $n \geq 2$ (the statement is trivial for $n=1$ ). Let $J_{n}$ be the indicator of the event that there is a strict maximum among the $n$ first variables, meaning that

$$
J_{n}=1_{\left\{\exists 1 \leq i \leq n: \forall 1 \leq j \leq n, j \neq i, T_{i}>T_{j}\right\}} .
$$

By exchangeability of the $n$ first variables we get that

$$
\mathrm{E}\left(I_{n}\right)=\frac{1}{n} \mathrm{E}\left(J_{n}\right) \leq \frac{1}{n} .
$$

Suppose now that the statement is true for $p \geq 1$ and let $2 \leq n_{1}<\ldots<n_{p+1}$ (if $n_{1}=1$, we can safely remove it). Again, by exchangeability of $\left(T_{1}, \ldots, T_{n_{1}}\right)$ we have

$$
\mathrm{E}\left(I_{n_{1}} \ldots I_{n_{p+1}}\right)=\frac{1}{n_{1}} \mathrm{E}\left(J_{n_{1}} I_{n_{2}} \ldots I_{n_{p+1}}\right) \leq \frac{1}{n_{1}} \mathrm{E}\left(I_{n_{2}} \ldots I_{n_{p+1}}\right),
$$

and the result follows by using the induction hypothesis.

Proof of Proposition 6.3. Let $\lambda>0$ to be specified later. By Chernoff's bound,

$$
\mathrm{P}\left(\mathfrak{R}_{n} \geq b \log n\right) \leq e^{-b \lambda \log n} \mathrm{E}\left[e^{\lambda R_{n}}\right] .
$$

Since $\Re_{n}=\sum_{k=1}^{n} I_{k}$ and the $I_{k}$ 's are $\{0,1\}$ valued random variables, we get

$$
e^{\lambda \Re_{n}}=\prod_{k=1}^{n}\left(1+\left[e^{\lambda}-1\right] I_{k}\right)=1+\sum_{\substack{J \subseteq\{1, \ldots, n\} \\ J \neq \varnothing}}\left[e^{\lambda}-1\right]^{|J|} \prod_{j \in J} I_{j} .
$$

By taking the expectation and using Proposition 6.2, we get

$$
\mathrm{E}\left[e^{\lambda \Re_{n}}\right] \leq \prod_{k=1}^{n}\left(1+\left[e^{\lambda}-1\right] \frac{1}{k}\right) \leq \exp \left(\left[e^{\lambda}-1\right][1+o(1)] \log n\right),
$$

where the $o(1)$ holds as $n \rightarrow \infty$. Finally, we obtain

$$
\mathrm{P}\left(\mathfrak{R}_{n} \geq b \log n\right) \leq \exp \left(\left\{\left[e^{\lambda}-1\right][1+o(1)]-b \lambda\right\} \log n\right),
$$

which concludes the proof.

Proof of Proposition 6.1. We will use notations from the previous subsection during the proof, and sometimes write $\mathfrak{R}(n)$ instead of $\mathfrak{R}_{n}$ for the sake of readability. We consider the events $A_{n}^{(i)}$ for $1 \leq i \leq 11$ and conclude by combining the results obtained in each cases. Along the proof we will use that, by (1.8), there exists $c_{1}, c_{2}>0$ such that for all $m \in \mathbb{N}$,

$$
1-c_{1} m^{-\gamma} \leq \mathbb{P}\left(T_{1} \leq m\right) \leq 1-c_{2} m^{-\gamma} \leq \exp \left(-c_{2} m^{-\gamma}\right) .
$$

Case $i=1$. Assume first that $\gamma \leq 1$, so that $\kappa=\frac{\gamma}{4}$. Since

$$
N^{\left(1+\frac{\gamma}{4}\right) \frac{2}{\gamma}}=o\left(n^{\left.1-\frac{\gamma}{4(\gamma+2)}\right)}\right. \text {, }
$$

one has $\lim _{n \rightarrow \infty} \mathbb{P}\left(A_{n}^{(1)}\right)=0$ from Proposition 1.1. Assume now that $\gamma>1$. Then,

$$
N^{2(1+\kappa)}= \begin{cases}o\left(n^{1-\frac{2-\gamma}{4(2+\gamma)}}\right) & \text { if } \gamma \in(1,2) \\ o\left(n^{1+\frac{2 \gamma-1}{2(\gamma+2)}}\right) & \text { if } \gamma \geq 2,\end{cases}
$$

and again $\lim _{n \rightarrow \infty} \mathbb{P}\left(A_{n}^{(1)}\right)=0$ from Proposition 1.1. 
Case $i=2$. Note that, by (6.13),

(6.16)

$$
\mathbb{P}\left(A_{n}^{(2)}\left(\varepsilon_{0}\right)\right)=\mathbb{P}\left(T_{1} \leq \varepsilon_{0}^{\frac{1}{2 \gamma}} N^{1 / \gamma}\right)^{\varepsilon_{0} N} \geq\left(1-\frac{c_{1}}{\varepsilon_{0}^{\frac{1}{2}} N}\right)^{\varepsilon_{0} N}=e^{-c_{1} \varepsilon_{0}^{1 / 2}}+o(1) \quad n \rightarrow \infty .
$$

We obtain $\lim _{\varepsilon_{0} \rightarrow 0} \liminf _{n \rightarrow \infty} \mathbb{P}\left(A_{n}^{(2)}\left(\varepsilon_{0}\right)\right)=1$.

Case $i=3$. Here we note that $N^{1 / \gamma}=o(n)$ when $\gamma \leq 1$ and $N=o(n)$ when $\gamma>1$, and we conclude using Proposition 1.1 that for all $\varepsilon_{0}>0$

$$
\lim _{n \rightarrow \infty} \mathbb{P}\left(A_{n}^{(3)}\left(\varepsilon_{0}\right)\right)=0 .
$$

Case $i=4$. By independence of the $T_{\ell}$ 's, one has

$$
\mathbb{P}\left(A_{n}^{(4)}(\delta)^{c}\right) \leq \mathbb{P}\left(T_{1}<\mathcal{T}_{0}(\varepsilon) \vee \delta N^{\frac{1}{\gamma}}\right)^{N} .
$$

Therefore,

$$
\mathbb{P}\left(A_{n}^{(4)}(\delta)^{c}\right) \leq \exp \left(-c\left(N \mathcal{T}_{0}(\varepsilon)^{-\gamma} \wedge \delta^{-\gamma}\right)\right),
$$

from which we deduce that for all $\varepsilon>0$

$$
\lim _{\delta \rightarrow 0} \liminf _{n \rightarrow \infty} \mathbb{P}\left(A_{n}^{(4)}(\delta)\right)=1 .
$$

Case $i=5$. Recall the definitions of $R_{\varepsilon_{0}}(n)$ and $T_{k}^{*}$ in (3.2) and (3.4). We get

(6.21) $\mathbb{P}\left(A_{n}^{(5)}\left(\varepsilon_{0}, \varepsilon\right)^{c}\right) \leq \mathbb{P}\left(\forall \ell \leq \varepsilon_{0} N, T_{\ell} \leq \mathcal{T}_{0}(\varepsilon) \vee \varepsilon_{0}^{\frac{3}{2 \gamma}} N^{\frac{1}{\gamma}}\right) \leq \mathbb{P}\left(T_{1} \leq \mathcal{T}_{0}(\varepsilon) \vee \varepsilon_{0}^{\frac{3}{2 \gamma}} N^{\frac{1}{\gamma}}\right)^{\varepsilon_{0} N}$.

By using again (6.13), we obtain

$$
\mathbb{P}\left(A_{n}^{(5)}\left(\varepsilon_{0}, \varepsilon\right)^{c}\right) \leq \exp \left(-c_{2}\left(\varepsilon_{0} \mathcal{T}_{0}(\varepsilon)^{-\gamma} N \wedge \varepsilon_{0}^{-1 / 2}\right)\right),
$$

from which we get for all $\varepsilon>0$

$$
\lim _{\varepsilon_{0} \rightarrow 0} \liminf _{n \rightarrow \infty} \mathbb{P}\left(A_{n}^{(5)}\left(\varepsilon_{0}, \varepsilon\right)\right)=1 .
$$

Case $6 \leq i \leq 8$. Let us first prove that for $\varepsilon_{0}>0$,

$$
\lim _{n \rightarrow \infty} \mathbb{P}\left(A_{n}^{(8)}\left(\varepsilon_{0}\right)\right)=1 .
$$

Indeed, since $\left|R\left(1, N / \varepsilon_{0}\right)\right|=\mathfrak{R}\left(N / \varepsilon_{0}\right)$, it is enough to prove that

$$
\mathbb{P}\left(\Re_{n} \leq(\log n)^{2}\right) \rightarrow 1, \quad n \rightarrow \infty,
$$

which can be easily deduced from Proposition 6.3. We now deal with the event corresponding to $i=7$. Fix $\varepsilon_{0}, \varepsilon>0$, and note that

$$
\begin{aligned}
\mathbb{P}\left(A_{n}^{(7)}\left(\varepsilon_{0}, \varepsilon\right)^{c}\right) \leq \mathbb{P}\left(A_{n}^{(8)}\left(\varepsilon_{0}\right)^{c}\right)+\mathbb{P}\left(A_{n}^{(5)}\left(\varepsilon_{0}, \varepsilon\right)^{c}\right) \\
\quad+\mathbb{P}\left(\exists k \leq\left[\log \left(N / \varepsilon_{0}\right)\right]^{2}: \varepsilon_{0}^{\frac{3}{2 \gamma}} N^{\frac{1}{\gamma}} \leq T_{k}^{*} \leq h\left(f_{k}, L_{k}, \alpha(\varepsilon)\right), i(k) \leq N / \varepsilon_{0}\right) .
\end{aligned}
$$

By a union bound we are left to prove that

$$
p_{k}\left(n, \varepsilon_{0}\right)=o\left([\log n]^{-2}\right),
$$

where

$$
p_{k}\left(n, \varepsilon_{0}, \varepsilon\right):=\mathbb{P}\left(\varepsilon_{0}^{\frac{3}{2 \gamma}} N^{\frac{1}{\gamma}} \leq T_{k}^{*} \leq h\left(f_{k}, L_{k}, \alpha(\varepsilon)\right), i(k) \leq N / \varepsilon_{0}\right) .
$$


From (3.65) in the proof of Lemma 3.8, we have for $n$ large enough,

$$
p_{k}\left(n, \varepsilon_{0}, \varepsilon\right) \leq \mathbb{P}\left(f_{k}^{L} \geq C\left(\varepsilon_{0}, \varepsilon\right) N^{\frac{1}{2 \gamma}}, T_{k}^{*} \geq \varepsilon_{0}^{\frac{3}{2 \gamma}} N^{\frac{1}{\gamma}}, i(k) \leq N / \varepsilon_{0}\right) .
$$

Let us first show that

$$
\mathbb{P}\left(f_{k}>A, T_{k}^{*} \geq \varepsilon_{0}^{\frac{3}{2 \gamma}} N^{\frac{1}{\gamma}}\right) \leq C\left[A \wedge \varepsilon_{0}^{\frac{3}{\gamma}} N^{\frac{2}{\gamma}}\right]^{-1} .
$$

To this end, our first ingredient is the following upper bound, which holds for $u \in(0,1)$ and $k \in \mathbb{N}$ :

$$
\mathbb{P}\left(\frac{T_{k}^{*}}{T_{k+1}^{*}} \geq 1-u\right) \leq C u .
$$

We show this inequality at the end of the proof, in order not to disrupt the main line of ideas. We will also use the following elementary bound:

$$
f(u)=\frac{u}{\sin (u)} \leq \frac{C}{\pi-u}, \quad u \in(0, \pi) .
$$

Coming back to (6.30), we have by using (6.32),

$$
\begin{aligned}
\mathbb{P}\left(f_{k}>A, T_{k}^{*} \geq \varepsilon_{0}^{\frac{3}{2 \gamma}} N^{\frac{1}{\gamma}}\right) & \leq \mathbb{P}\left(\frac{T_{k-1}^{*}}{T_{k}^{*}}\left[1+\frac{C}{\left(T_{k}^{*}\right)^{2}}\right] \geq 1-\frac{C}{A}, T_{k}^{*} \geq \varepsilon_{0}^{\frac{3}{2 \gamma}} N^{\frac{1}{\gamma}}\right) \\
& \leq \mathbb{P}\left(\frac{T_{k-1}^{*}}{T_{k}^{*}} \geq 1-\frac{C}{A}-\frac{C}{\varepsilon_{0}^{\frac{3}{\gamma}} N^{\frac{2}{\gamma}}}\right),
\end{aligned}
$$

and we obtain (6.30) thanks to (6.31). In view of (6.27), we choose $A=A_{n}=[\log n]^{3}$. For $k \geq 1$, from (6.29),

$p_{k}\left(n, \varepsilon_{0}, \varepsilon\right) \leq \mathbb{P}\left(f_{k} \geq A_{n}, T_{k}^{*} \geq \varepsilon_{0}^{\frac{3}{2 \gamma}} N^{\frac{1}{\gamma}}\right)+\mathbb{P}\left(L_{k} \geq C \frac{\log n}{\log \log n}, T_{k}^{*} \geq \varepsilon_{0}^{\frac{3}{2 \gamma}} N^{\frac{1}{\gamma}}, i(k) \leq N / \varepsilon_{0}\right)$.

Using (6.30), the first term in the sum above is $o\left[(\log n)^{-2}\right]$. We now deal with the second one. On the corresponding event, we have

$$
\operatorname{card}\left\{1 \leq j \leq N / \varepsilon_{0}: T_{j} \geq \alpha \varepsilon_{0}^{\frac{3}{2 \gamma}} N^{\frac{1}{\gamma}}\right\} \geq C \frac{\log n}{\log \log n} .
$$

Furthermore, the random variable in the l.h.s. of the inequality follows a binomial law with parameters $N / \varepsilon_{0}$ and $\mathbb{P}\left(T_{1} \geq \alpha \varepsilon_{0}^{\frac{3}{2 \gamma}} N^{\frac{1}{\gamma}}\right) \leq \alpha^{-\gamma} \varepsilon_{0}^{-3 / 2} N^{-1}$ (up to a harmless constant). By using a standard binomial-to-Poisson approximation (cf. end of the proof)

$$
\mathbb{P}(\operatorname{Bin}(\ell, q) \geq m) \leq\left(\frac{q \ell}{m}\right)^{m} e^{m-q \ell}, \quad \ell \in \mathbb{N}, q \in(0,1), m \in \mathbb{N}: q \ell<m,
$$

with $q=\varepsilon_{0}^{-3 / 2} N^{-1}, \ell=N / \varepsilon_{0}$ and $m=C \log n / \log \log n$, we get that

$$
\mathbb{P}((6.35))=o\left(n^{-C / 2}\right),
$$

which is enough to conclude. From what precedes we finally obtain that for all $\varepsilon_{0}, \varepsilon>0$,

$$
\lim _{n \rightarrow \infty} \mathbb{P}\left(A_{n}^{(7)}\left(\varepsilon_{0}, \varepsilon\right)^{c}\right)=0 .
$$

The event corresponding to $i=6$ can be readily treated with the same idea, since the $N^{\frac{1}{2 \gamma}}$ in (6.29) is less than $\exp \left(n^{\frac{\gamma}{2(\gamma+2)}}\right)$. Finally,

$$
\lim _{n \rightarrow \infty} \mathbb{P}\left(A_{n}^{(6)}\left(\varepsilon_{0}, \varepsilon\right)^{c} \cup A_{n}^{(7)}\left(\varepsilon_{0}, \varepsilon\right)^{c}\right)=0 .
$$


Case $9 \leq i \leq 10$. From the almost-sure convergence in Proposition 2.1, we readily get that for any choice of $\delta, \varepsilon_{0}, \varepsilon, \eta$,

$$
\lim _{n \rightarrow \infty} \mathbb{P}\left(A_{n}^{(9)}(\delta) \cap A_{n}^{(10)}\left(\varepsilon_{0}, \varepsilon, \eta\right)\right)=1 .
$$

Case $i=11$. Note that by (6.13),

$$
\mathbb{P}\left(A_{n}^{(11)}\left(\varepsilon_{0}\right)\right)=\mathbb{P}\left(T_{1} \leq \frac{N^{1 / \gamma}}{\varepsilon_{0}}\right)^{N} \geq e^{-c_{1} \varepsilon_{0}^{\gamma}}+o(1) \quad n \rightarrow \infty .
$$

We obtain $\lim _{\varepsilon_{0} \rightarrow 0} \liminf \operatorname{in}_{n \rightarrow \infty} \mathbb{P}\left(A_{n}^{(11)}\left(\varepsilon_{0}\right)\right)=1$.

Proof of (6.31). By writing $1+v=(1-u)^{-1}$ for convenience, one has

$$
\mathbb{P}\left(T_{k+1}^{*} \leq(1+v) T_{k}^{*} \mid T_{k}^{*}=\ell\right)= \begin{cases}0 & \text { if } v<1 / \ell \\ \mathbb{P}\left(T_{1} \leq\lfloor(1+v) \ell\rfloor \mid T_{1}>\ell\right) & \text { otherwise. }\end{cases}
$$

From our assumption on the tail of $T_{1}$ and with the help of a standard comparison between series and integrals, the probability in the second case is bounded from above by

$$
C \ell^{\gamma} \sum_{n=\ell+1}^{\lfloor(1+v) \ell\rfloor} n^{-(1+\gamma)} \leq C \ell^{\gamma} \int_{\ell}^{\lfloor(1+v) \ell\rfloor-1} t^{-(1+\gamma)} \mathrm{d} t .
$$

In turn, the integral above is controlled by

$$
\int_{\ell}^{(1+v) \ell} t^{-(1+\gamma)} \mathrm{d} t=C\left[1-(1+v)^{-\gamma}\right] \ell^{-\gamma} \leq C u \ell^{-\gamma}
$$

which completes the proof of (6.31).

Proof of (6.36). By Chernoff's bound, one has for $x>0$,

$$
\mathbb{P}(\operatorname{Bin}(\ell, q) \geq m) \leq e^{-x m} \mathbb{E}\left(e^{x \operatorname{Ber}(q)}\right)^{\ell} \leq \exp \left(-x m+q\left(e^{x}-1\right) \ell\right) .
$$

Since $m>q \ell$, we may choose $x=\log (m /(q \ell))$ to minimize the r.h.s. in the line above and get the result.

6.2. Conclusion: proof of Theorem 2.2. To prove Theorem 2.2, we establish that for all $u \in \mathbb{R}$ which is a continuity point of the distribution function of $F$,

$$
\lim _{n \rightarrow \infty} \mathbb{P}\left(F_{n} \leq u\right)=\mathbb{P}(F \leq u) .
$$

By (2.36) all real numbers are continuity points of $F$. Moreover since $F_{n}$ is positive, we only have to prove (6.46) for $u>0$.

We start with the upper bound in (6.46)

$$
\limsup _{n \rightarrow \infty} \mathbb{P}\left(F_{n} \leq u\right) \leq \mathbb{P}(F \leq u) .
$$

Fix $\theta>0$ that we will let go to 0 only at the very end of the proof. Fix also $\varepsilon, \eta>0$. From Proposition 6.1 , there exists $\delta>0$ and $\varepsilon_{1}(\delta)$ so that for $\varepsilon_{0}<\varepsilon_{1}(\delta)$

$$
\liminf _{n} \mathbb{P}\left(\Omega_{n}\left(\delta, \varepsilon_{0}, \varepsilon, \eta\right)\right)>1-\theta .
$$

Fix $\varepsilon_{0}<\varepsilon_{1}(\delta)$ small enough so that the conclusion of Proposition 5.1 is satisfied. Thus we obtain that for $n$ large enough

$$
\mathbb{P}\left(F_{n} \leq u\right) \leq \mathbb{P}\left(\min _{\ell \in \mathcal{R}_{\varepsilon_{0}}(n)} G_{n}^{\beta-\varepsilon}(\ell) \leq u+\eta, \Omega_{n}\right)+\mathbb{P}\left(\Omega_{n}^{c}\right) .
$$


From Proposition 6.1 and the choices of $\delta$ and $\varepsilon_{0}, \mathbb{P}\left(\Omega_{n}^{c}\right)<\theta$ for $n$ large enough. Thus we just have to focus on the first term in the last equation. We introduce for $n, \ell \geq 1$ and $\beta>0$, the random variable

$$
\widetilde{G}_{n}^{\beta}(\ell)=\frac{\lambda(\beta)(\ell-1)}{N}+\frac{\pi^{2} n}{2 T_{\ell}^{2} N}=\psi^{\lambda(\beta)}\left(\frac{\ell}{N}, \frac{T_{\ell}}{N^{1 / \gamma}}\right) .
$$

We replace $G$ by $\widetilde{G}$ in (6.49) and control the probability that both processes are not close (6.51)

$$
\begin{aligned}
\mathbb{P}\left(\min _{\ell \in \mathcal{R}_{\varepsilon_{0}}(n)} G_{n}^{\beta-\varepsilon}(\ell) \leq u+\eta, \Omega_{n}\right) & \\
& \leq \mathbb{P}\left(\min _{\ell \in \mathcal{R}} \widetilde{G}_{n}^{\beta-\varepsilon}(\ell)<u+2 \eta, \Omega_{n}\right)+\mathbb{P}\left(\max _{\ell \in \mathcal{R}_{\varepsilon_{0}}(n)}\left|\widetilde{G}_{n}^{\beta-\varepsilon}(\ell)-G_{n}^{\beta-\varepsilon}(\ell)\right| \geq \eta, \Omega_{n}\right) .
\end{aligned}
$$

The first term in the sum gives the main contribution. Let us first prove that the second one is zero for $n$ large enough. For $\ell \geq 1$ we define

$$
\Delta_{1}(n, \ell):=\frac{\ell-1}{N}|\lambda(\beta-\varepsilon)-\lambda(\ell-1, \beta-\varepsilon)|
$$

and

$$
\Delta_{2}(n, \ell):=\frac{n}{N}\left|g\left(T_{\ell}\right)-\frac{\pi^{2}}{2 T_{\ell}^{2}}\right|
$$

so that

$$
\max _{\ell \in \mathcal{R}_{\varepsilon_{0}}(n)}\left|\widetilde{G}_{n}^{\beta-\varepsilon}(\ell)-G_{n}^{\beta-\varepsilon}(\ell)\right| \leq \max _{\ell \in \mathcal{R}_{\varepsilon_{0}}(n)} \Delta_{1}(n, \ell)+\max _{\ell \in \mathcal{R}_{\varepsilon_{0}}(n)} \Delta_{2}(n, \ell) .
$$

We first deal with $\Delta_{2}$. According to (3.14) there exists some $C>0$ such that for all $\ell$, $\left|g\left(T_{\ell}\right)-\frac{\pi^{2}}{2 T_{\ell}^{2}}\right| \leq \frac{C}{T_{\ell}^{4}}$. We can thus deduce that

$$
\mathbb{P}\left(\max _{\ell \in \mathcal{R}_{\varepsilon_{0}}(n)} \Delta_{2}(n, \ell) \geq \eta, \Omega_{n}\right) \leq \mathbb{P}\left(\frac{C n}{N\left(\varepsilon_{0}^{\frac{3}{2 \gamma}} N^{1 / \gamma}\right)^{4}} \geq \eta, A_{n}^{(5)}\left(\varepsilon_{0}, \varepsilon\right)\right),
$$

and this last term is 0 for $n$ large enough. We turn to the control of $\Delta_{1}$. For $n \in \mathbb{N}$,

$$
\mathbb{P}\left(\max _{\ell \in \mathcal{R}_{\varepsilon_{0}}(n)} \Delta_{1}(n, \ell) \geq \eta, \Omega_{n}\right) \leq \mathbb{P}\left(\max _{\ell \in \mathcal{R}_{\varepsilon_{0}}(n)} \Delta_{1}(n, \ell) \geq \eta, A_{n}^{(10)}\left(\varepsilon_{0}, \varepsilon, \eta\right)\right),
$$

and again the last term is 0 for $n$ large enough. Let us come back to the first term in (6.51), $\mathbb{P}\left(\min _{\ell \in \mathcal{R}} \widetilde{G}_{n}^{\beta-\varepsilon}(\ell)<u+2 \eta, \Omega_{n}\right)$. As $\ell$ ranges $\mathcal{R}$ we may write

$$
\min _{\ell \in \mathcal{R}} \widetilde{G}_{n}^{\beta-\varepsilon}(\ell)=\Psi^{\lambda(\beta-\varepsilon)}\left(\Pi_{N}\right) .
$$

We thus obtain

$$
\begin{aligned}
\mathbb{P}\left(\min _{\ell \in \mathcal{R}} \widetilde{G}_{n}^{\beta-\varepsilon}(\ell)<u+2 \eta, \Omega_{n}\right) & \leq \mathbb{P}\left(\Psi^{\lambda(\beta-\varepsilon)}\left(\Pi_{N}\right)<u+2 \eta, A_{n}^{(11)}\left(\varepsilon_{0}\right)\right) \\
& \leq \mathbb{P}\left(\Psi_{K}^{\lambda(\beta-\varepsilon)}\left(\Pi_{N}\right)<u+2 \eta\right),
\end{aligned}
$$

where $K:=A_{u+2 \eta}^{\lambda(\beta-\varepsilon)} \cap\left\{y \leq 1 / \varepsilon_{0}\right\}$ with the set $A$ defined in (2.22). As $K$ is compact, Proposition 2.4 and Lemma 2.6 assure that

$$
\mathbb{P}\left(\Psi_{K}^{\lambda(\beta-\varepsilon)}\left(\Pi_{N}\right)<u+2 \eta\right) \rightarrow \mathbb{P}\left(\Psi_{K}^{\lambda(\beta-\varepsilon)}(\Pi)<u+2 \eta\right)
$$


when $n$ goes to infinity (we recall that $\Psi_{K}^{\lambda(\beta-\varepsilon)}(\Pi)$ is continuous). Using that $\Psi^{\lambda(\beta-\varepsilon)} \leq$ $\Psi_{K}^{\lambda(\beta-\varepsilon)}$ we obtain

$$
\begin{aligned}
\mathbb{P}\left(\Psi_{K}^{\lambda(\beta-\varepsilon)}(\Pi)<u+2 \eta\right) & \leq \mathbb{P}\left(\Psi^{\lambda(\beta-\varepsilon)}(\Pi)<u+2 \eta\right) \\
& =\mathbb{P}\left(F^{\beta-\varepsilon}<u+2 \eta\right) .
\end{aligned}
$$

Finally, we have proven

$$
\limsup _{n \rightarrow \infty} \mathbb{P}\left(F_{n} \leq u\right) \leq \mathbb{P}\left(F^{\beta-\varepsilon}<u+2 \eta\right)+\theta .
$$

As $u \mapsto \mathbb{P}\left(F^{\beta-\varepsilon} \leq u\right)$ is right-continuous,

$$
\lim _{\eta \rightarrow 0} \mathbb{P}\left(F^{\beta-\varepsilon}<u+2 \eta\right)=\mathbb{P}\left(F^{\beta-\varepsilon} \leq u\right) .
$$

From Lemma 2.7, $F^{\beta-\varepsilon} \nearrow F^{\beta}$ almost surely when $\varepsilon$ goes to 0 so that

$$
\mathbb{P}\left(F^{\beta-\varepsilon} \leq u\right) \rightarrow \mathbb{P}\left(F^{\beta} \leq u\right) \quad \varepsilon \rightarrow 0 .
$$

Finally,

$$
\limsup _{n \rightarrow \infty} \mathbb{P}\left(F_{n} \leq u\right) \leq \mathbb{P}\left(F^{\beta} \leq u\right)+\theta,
$$

and, as $\theta$ can be chosen arbitrarily small, we obtain the upper bound

$$
\limsup _{n \rightarrow \infty} \mathbb{P}\left(F_{n} \leq u\right) \leq \mathbb{P}\left(F^{\beta} \leq u\right) .
$$

We turn now to the lower bound in (6.46):

$$
\liminf _{n \rightarrow \infty} \mathbb{P}\left(F_{n} \leq u\right) \geq \mathbb{P}(F \leq u),
$$

or, equivalently,

$$
\limsup _{n \rightarrow \infty} \mathbb{P}\left(F_{n}>u\right) \leq \mathbb{P}(F>u) .
$$

The proof works essentially in the same way as for the upper bound. Again fix $\theta, \varepsilon, \eta>0$ and, using Proposition $6.1, \delta>0$ and $\varepsilon_{1}(\delta)$ so that for $\varepsilon_{0}<\varepsilon_{1}(\delta)$

$$
\liminf _{n} \mathbb{P}\left(\Omega_{n}\left(\delta, \varepsilon_{0}, \varepsilon, \eta\right)\right)>1-\theta .
$$

We choose $\varepsilon_{0}<\varepsilon_{1}(\delta)$ small enough so that

(1) the conclusion of Proposition 4.1 is satisfied;

(2) the following inequality holds

$$
\frac{\lambda(\beta)}{\varepsilon_{0}}>2 \lambda(\beta)+\frac{\pi^{2}}{2 \delta^{2}} .
$$

Using Proposition 4.1, for $n$ large enough,

$$
\begin{aligned}
\mathbb{P}\left(F_{n}>u\right) & \leq \mathbb{P}\left(\min _{1<\ell \leq N^{1+\kappa}} G_{n}^{\beta}(\ell)>u-\eta, \Omega_{n}\right)+\mathbb{P}\left(\Omega_{n}^{c}\right) \\
& \leq \mathbb{P}\left(\min _{\ell \in \mathcal{R}_{\varepsilon_{0}}(n)} G_{n}^{\beta}(\ell)>u-\eta, \Omega_{n}\right)+\mathbb{P}\left(\Omega_{n}^{c}\right) \\
& \leq \mathbb{P}\left(\min _{\ell \in \mathcal{R}_{\varepsilon_{0}}(n)} \widetilde{G}_{n}^{\beta}(\ell)>u-2 \eta, \Omega_{n}\right)+\mathbb{P}\left(\max _{\ell \in \mathcal{R}_{\varepsilon_{0}}(n)}\left|\widetilde{G}_{n}^{\beta}(\ell)-G_{n}^{\beta}(\ell)\right| \geq \eta, \Omega_{n}\right)+\mathbb{P}\left(\Omega_{n}^{c}\right) .
\end{aligned}
$$


The second term in this last equation is treated exactly in the same way as the second term in (6.51) and is thus zero for $n$ large enough. The third one is smaller than $\theta$ by (6.68) for $n$ large enough. We thus focus on the first one. The choice of $\varepsilon_{0}$ in (6.69) implies that

$$
\Omega_{n} \subset\left\{\operatorname{argmin} \widetilde{G}_{n}<N / \varepsilon_{0}\right\} .
$$

Indeed, as $\Omega_{n} \subset A_{n}^{(4)}(\delta)$, it holds that, on $\Omega_{n}$,

$$
\min _{N \leq \ell \leq 2 N} \widetilde{G}_{n}^{\beta}<2 \lambda(\beta)+\frac{\pi^{2}}{2 \delta^{2}}<\frac{\lambda(\beta)}{\varepsilon_{0}}<\min _{\ell>N / \varepsilon_{0}} \widetilde{G}_{n}^{\beta}(\ell) .
$$

Therefore, for any compact set $K$ in $E$,

$$
\begin{aligned}
\mathbb{P}\left(\min _{\ell \in \mathcal{R}_{\varepsilon_{0}}(n)} \widetilde{G}_{n}^{\beta}(\ell)>u-2 \eta, \Omega_{n}\right) & =\mathbb{P}\left(\min _{\ell \in \mathcal{R}} \widetilde{G}_{n}^{\beta}(\ell)>u-2 \eta, \Omega_{n}\right) \\
& =\mathbb{P}\left(\Psi^{\lambda(\beta)}\left(\Pi_{N}\right)>u-2 \eta, \Omega_{n}\right) \\
& \leq \mathbb{P}\left(\Psi_{K}^{\lambda(\beta)}\left(\Pi_{N}\right)>u-2 \eta\right) .
\end{aligned}
$$

By Lemma 2.6, $\mathbb{P}\left(\Psi_{K}^{\lambda(\beta)}\left(\Pi_{N}\right)>u-2 \eta\right)$ converges to $\mathbb{P}\left(\Psi_{K}^{\lambda(\beta)}(\Pi)>u-2 \eta\right)$ when $N$ goes to infinity. Finally,

$$
\limsup _{n \rightarrow \infty} \mathbb{P}\left(F_{n}>u\right) \leq \mathbb{P}\left(\Psi_{K}^{\lambda(\beta)}(\Pi)>u-2 \eta\right)+\theta .
$$

By letting $K$ increase to $E$, we obtain

$$
\limsup _{n \rightarrow \infty} \mathbb{P}\left(F_{n}>u\right) \leq \mathbb{P}\left(\Psi^{\lambda(\beta)}(\Pi)>u-2 \eta\right)+\theta,
$$

and we conclude as for the upper bound by letting $\eta$ and $\theta$ go to 0 .

\section{Appendix A. Proof of Proposition 3.3}

The proof is divided into several steps. In the following, we partition $\tau$ into $p$ disjoint subsets $\tau^{(i)}=\tau_{i}+\tau_{p} \mathbb{Z}$, for $0 \leq i<p$.

Step 1. Decomposition of the probability. We first consider the event $\left\{\sigma>n, S_{n} \in\right.$ $\tau\}$ instead of $\{\sigma>n\}$ and will come back to the original event at the final step. By decomposing according to the visits to $\tau$ and by using the Markov property, we obtain

$$
\mathrm{P}\left(\sigma>n, S_{n} \in \tau\right)=\sum_{m=1}^{n} \sum_{0<u_{1}<\ldots<u_{m}=n} \sum_{x_{1}, \ldots, x_{m}} \prod_{i=1}^{m}\left(q_{x_{i-1}, x_{i}}\left(u_{i}-u_{i-1}\right) e^{-\beta}\right),
$$

where $u_{0}=0, x_{0}=0, x_{1}, \ldots, x_{m} \in \mathbb{Z} / p \mathbb{Z}$, and the $q_{i j}(n)$ 's are a slight modification of the ones defined in (3.9), namely

$$
q_{i j}(n)=\mathrm{P}_{\tau_{i}}\left(S_{k} \notin \tau, 1 \leq k<n, S_{n} \in \tau^{(j)}\right), \quad i, j \in \mathbb{Z} / p \mathbb{Z}, \quad n \in \mathbb{N} .
$$

It will be helpful later in the proof to know the asymptotic behaviour of $q_{i j}(n)$, as $n \rightarrow \infty$ :

$$
\lim \frac{1}{n} \log q_{i j}(n)=-g\left(t_{i j}\right), \quad n \rightarrow \infty,
$$

where $g$ has been defined in (3.14) and $t_{i j}$ in (3.11).

Step 2. Definition of $\phi\left(\beta ; t_{1}, \ldots, t_{p}\right)$. Recall the definition of $Q_{i j}(\phi)$ in (3.10) (with $q_{i j}$ defined in (A.2)), which is now restricted to $i, j \in \mathbb{Z} / p \mathbb{Z}$. For all $i, j, Q_{i j}(\phi)$ is finite and increasing on $\left[0, g\left(t_{i j}\right)\right)$, infinite on $\left[g\left(t_{i j}\right), \infty\right)$ and its limit at $g\left(t_{i j}\right)$ is infinite, by 
Proposition 3.1. Let $\Lambda(\phi)$ be the Perron-Frobenius eigenvalue of $Q(\phi)$, defined as infinity when one of the entry is infinite, that is for $\phi \geq \min g\left(t_{i j}\right)=g\left(t_{\max }\right)$. We recall that

$$
\Lambda(\phi)=\sup _{v \neq 0} \min _{i} \frac{(Q(\phi) v)_{i}}{v_{i}} .
$$

From what precedes, $\Lambda$ is increasing on $\left[0, g\left(t_{\max }\right)\right)$ and tends to $\infty$ as $\phi \nearrow g\left(t_{\max }\right)$. Therefore, the equation

$$
\Lambda(\phi)=\exp (\beta)
$$

has a unique positive solution on this interval, that we denote by $\phi\left(\beta ; t_{1}, \ldots, t_{p}\right)$. As $\phi\left(\beta ; t_{1}, \ldots, t_{p}\right) \in\left[0, g\left(t_{\max }\right)\right)$, this proves the second inequality in $(3.20)$. In the sequel of the proof, for the sake of conciseness, we use the notation $\phi(\beta)=\phi\left(\beta ; t_{1}, \ldots, t_{p}\right)$. Coming back to (A.1), we get

$$
\text { r.h.s(A.1) }=e^{-\phi(\beta) n} \sum_{m=1}^{n} \sum_{0<u_{1}<\ldots<u_{m}=n} \sum_{x_{1}, \ldots, x_{m}} \prod_{i=1}^{m}\left(q_{x_{i-1}, x_{i}}\left(u_{i}-u_{i-1}\right) e^{-\beta+\phi(\beta)\left(u_{i}-u_{i-1}\right)}\right) \text {. }
$$

Step 3. Spectral decomposition and a first upper bound. The key idea in this step is a spectral decomposition, which is a technique used also in the context of the parabolic Anderson model, see [13, Section 2.2.1]. Let us define a matrix $Q^{\beta}$ by

$$
Q_{i j}^{\beta}=Q_{i j}(\phi(\beta)) e^{-\beta}, \quad i, j \in \mathbb{Z} / p \mathbb{Z},
$$

which is symmetric (by symmetry of the simple random walk) with positive entries. From what precedes, its top eigenvalue is one. Therefore, we may denote by $1=\lambda_{0} \geq \lambda_{1} \geq$ $\ldots \geq \lambda_{p-1}$ its eigenvalues by decreasing order (with possible repetitions), with $\left|\lambda_{k}\right|<1$ for $k>1$ (Theorem 1.1 in Seneta [17]) and $\left(\nu_{i}\right)_{0 \leq i<p}$ an associated basis of orthonormal left eigenvectors. Note that for all $0 \leq x<p$, we have $\delta_{x}=\sum_{i=0}^{p-1} \nu_{i}(x) \nu_{i}$, that is the element of $\mathbb{R}^{p}$ which is 1 at coordinate $x$ and 0 elsewhere. Let us now define, for $0 \leq a<p$,

$$
\mathcal{Z}_{n}(a)=\sum_{m=1}^{n} \sum_{x_{0}=a, x_{1}, \ldots, x_{m}} \prod_{i=1}^{m} Q_{x_{i-1}, x_{i}}^{\beta} .
$$

By removing the condition $\left\{u_{m}=n\right\}$ in (A.6), we get the upper bound

$$
\text { r.h.s.(A.6) } \leq e^{-\phi(\beta) n} \mathcal{Z}_{n}(0) \text {. }
$$

Moreover,

$$
\mathcal{Z}_{n}(0)=\left\langle\delta_{0}, \mathcal{Z}_{n}(\cdot)\right\rangle=\sum_{i=0}^{p-1} \sum_{j=0}^{p-1} \nu_{i}(0) \nu_{i}(j) \mathcal{Z}_{n}(j)
$$

(with the usual scalar product) which yields

$$
\mathcal{Z}_{n}(0)=\sum_{i=0}^{p-1} \sum_{j=0}^{p-1} \sum_{m=1}^{n} \nu_{i}(0) \nu_{i}(j) \sum_{x_{0}=j, x_{1} \ldots, x_{m}} \prod_{k=1}^{m} Q_{x_{k-1}, x_{k}}^{\beta} .
$$

By definition of the $\nu_{i}$ 's we get for $0 \leq i<p$,

$$
\sum_{j=0}^{p-1} \nu_{i}(j) \sum_{x_{0}=j, x_{1}, \ldots, x_{m}} \prod_{k=1}^{m} Q_{x_{k-1}, x_{k}}^{\beta}=\nu_{i}\left(Q^{\beta}\right)^{m} 1=\lambda_{i}^{m} \sum_{j=0}^{p-1} \nu_{i}(j),
$$


where 1 is the vector with all one. Therefore,

$$
\mathcal{Z}_{n}(0)=\sum_{i=0}^{p-1} \sum_{j=0}^{p-1} \sum_{m=1}^{n} \nu_{i}(0) \nu_{i}(j) \lambda_{i}^{m} \leq n \sum_{i=0}^{p-1} \sum_{j=0}^{p-1}\left|\nu_{i}(0)\right|\left|\nu_{i}(j)\right| \leq n p,
$$

where in the first inequality we use that $\left|\lambda_{i}\right| \leq 1$ and the triangular inequality, while in the second inequality we use the Cauchy-Schwarz inequality and the fact that

$$
\sum_{j=0}^{p-1} \nu_{i}(j)^{2}=\left\|\nu_{i}\right\|_{2}^{2}=1, \quad \sum_{i=0}^{p-1} \nu_{i}(0)^{2}=\left\|\delta_{0}\right\|_{2}^{2}=1 .
$$

Finally, we have obtained

$$
\mathrm{P}\left(\sigma>n, S_{n} \in \tau\right) \leq n p e^{-\phi(\beta) n} .
$$

Step 4. Lower bound in (3.15). The components of a Perron-Frobenius eigenvector being all positive (or all negative), we may consider the matrix $\left\{Q_{i j}^{\beta} \frac{\nu_{0}(j)}{\nu_{0}(i)}\right\}_{i j}$, which turns out to be stochastic. This actually defines a Markov renewal process $\rho$ on $\mathbb{Z} / p \mathbb{Z}$ (see e.g. Section VII.4 in Asmussen [2]) with law $\mathcal{P}_{\beta}$ determined by the kernel

$$
q_{i j}^{\beta}(n)=\exp (\phi(\beta) n-\beta) q_{i j}(n) \frac{\nu_{0}(j)}{\nu_{0}(i)}, \quad n \geq 1, \quad i, j \in \mathbb{Z} / p \mathbb{Z},
$$

and starting from state 0 . Therefore, we may write

$$
\begin{aligned}
& \mathrm{P}\left(\sigma>n, S_{n} \in \tau\right) \geq \mathrm{P}\left(\sigma>n, S_{n} \in \tau^{(0)}\right) \\
& =e^{-\phi(\beta) n} \sum_{m=1}^{n} \sum_{0<u_{1}<\ldots<u_{m}=n} \sum_{\begin{array}{c}
x_{1}, \ldots, x_{m-1} \\
x_{0}=x_{m}=0
\end{array}} \prod_{i=1}^{m}\left(q_{x_{i-1}, x_{i}}\left(u_{i}-u_{i-1}\right) e^{-\beta+\phi(\beta)\left(u_{i}-u_{i-1}\right)}\right) \\
& =e^{-\phi(\beta) n} \sum_{m=1}^{n} \sum_{0<u_{1}<\ldots<u_{m}=n} \sum_{\substack{x_{1}, \ldots, x_{m-1} \\
x_{0}=x_{m}=0}} \prod_{i=1}^{m} q_{x_{i-1}, x_{i}}^{\beta}\left(u_{i}-u_{i-1}\right) \\
& =e^{-\phi(\beta) n} \mathcal{P}_{\beta}\left(n \in \rho_{0}\right),
\end{aligned}
$$

where $\rho_{0}$ is the subset of $\rho$ formed by the Markov renewal points with state 0 . It turns out that it is a renewal process. By the second inequality in (3.20) (that we have already proven in Step 2) $q_{i j}^{\beta}$ decays exponentially in $n$ for all $i, j \in \mathbb{Z} / p \mathbb{Z}$. This implies (as the modulating Markov chain has finite state space) that the inter-arrival law of $\rho_{0}$ also decays exponentially in $n$, which implies integrability. Therefore, by the renewal theorem, $\mathcal{P}_{\beta}\left(n \in \rho_{0}\right)$ converges to some constant (that is the inverse of the mean inter-arrival time). This concludes this step.

Step 5. Proof of (3.20). The second inequality has already been established in Step 2, so let us prove the first inequality. A standard coupling argument yields

$$
\sum_{j} Q_{i j}(\phi)=\mathrm{E}_{\tau_{i}}\left(e^{\phi \theta_{1}}\right) \leq \mathrm{E}\left(e^{\phi \theta_{1}^{\max }}\right), \quad i \in \mathbb{Z} / p \mathbb{Z}
$$

where $\theta_{1}=\inf \left\{n \in \mathbb{N}: S_{n} \in \tau\right\}$ and $\theta_{1}^{\max }=\inf \left\{n \in \mathbb{N}: S_{n} \in t_{\max } \mathbb{Z}\right\}$. By Proposition 3.2 , we get that $\sum_{j} Q_{i j}\left(\phi\left(\beta, t_{\max }\right)\right)=e^{\beta}$. Thanks to Lemma A.1 below, it means that $\Lambda\left(\phi\left(\beta, t_{\max }\right)\right) \leq e^{\beta}$ and we get the desired bound, as $\Lambda$ is non-decreasing. 
Step 6. Final upper bound. We now conclude by removing the condition $\left\{S_{n} \in \tau\right\}$ in the upper bound. To this end, we decompose according to the last visit to $\tau$ before $n$ :

$$
\mathrm{P}\left(\sigma>n, S_{n} \notin \tau\right)=\sum_{m=0}^{n-1} \sum_{j=0}^{p-1} \mathrm{P}\left(\sigma>m, S_{m} \in \tau^{(j)}\right) \mathrm{P}_{\tau_{j}}\left(S_{k} \notin \tau, k \leq n-m\right) .
$$

By using Proposition 3.1, we get that there exists $C$ such that for all $0 \leq j<p$ and $n \in \mathbb{N}$,

$$
\mathrm{P}_{\tau_{j}}\left(S_{k} \notin \tau, k \leq n\right) \leq C e^{-\min g\left(t_{i j}\right) n}=C e^{-g\left(t_{\max }\right) n},
$$

provided $t_{i j}$ larger than $\mathcal{T}_{0}$. To deal with the values of $t_{i j}$ smaller than $\mathcal{T}_{0}$, we may use an explicit expression of the small-ball probability provided in Chapter XIV of Feller [9]. By using (3.20), we get

$$
\begin{aligned}
\mathrm{P}\left(\sigma>n, S_{n} \notin \tau\right) & \leq C \sum_{m=0}^{n} \sum_{j=0}^{p-1} \mathrm{P}\left(\sigma>m, S_{m} \in \tau^{(j)}\right) e^{-\phi(\beta)(n-m)} \\
& =C \sum_{m=0}^{n} \mathrm{P}\left(\sigma>m, S_{m} \in \tau\right) e^{-\phi(\beta)(n-m)} \\
& \leq C n^{2} p \exp (-\phi(\beta) n),
\end{aligned}
$$

where we have used (A.15) to go from the second to the last line.

Lemma A.1. If the sums over lines of a non-negative matrix $A$ are less than one, then its Perron-Frobenius eigenvalue is less than one.

Proof of Lemma A.1. Let $\lambda$ be an eigenvalue of $A$ and $v$ an associated eigenvector such that $v_{i_{*}}=\max _{i} v_{i}>0$. Then

$$
\lambda v_{i_{*}}=(A v)_{i_{*}} \leq \sum_{j} A_{i_{*}, j} v_{i_{*}} \leq v_{i_{*}}
$$

and that is enough to conclude as $v_{i_{*}}>0$.

\section{Appendix B. Proof of Theorem 2.8}

For simplicity, we only treat the case $T=1$ as the case of a general $T>0$ is similar. We use the standard two-step proof: convergence of the finite-dimensional marginal distributions and tightness.

Finite-dimensional marginal distributions. Let $k \in \mathbb{N}$ and $0 \leq s_{1}<\ldots<s_{k} \leq 1$. By the Portmanteau theorem, it is enough to prove convergence of the joint cumulative distribution function of $\left(F_{n}\left(s_{1}\right), \ldots, F_{n}\left(s_{k}\right)\right)$ to that of $\left(F\left(s_{1}\right), \ldots, F\left(s_{k}\right)\right)$. This is done by adapting what we have done for the one-dimensional distribution. For each coordinate $1 \leq i \leq k$ denote by $\Omega_{n}(i)$ the corresponding good environment, that is $\Omega_{\left\lfloor s_{i} n\right\rfloor}$, and consider $\bar{\Omega}_{n}:=\Omega_{n}(1) \cap \ldots \cap \Omega_{n}(k)$. By a union bound, Proposition 6.1 holds with $\bar{\Omega}_{n}$ instead of $\Omega_{n}$. From here, it is possible to follow the proof in Section 6.2, with the cylinders ] $\left.-\infty, u_{1}\right] \times$ $\ldots \times]-\infty, u_{k}$ ] instead of $\left.]-\infty, u\right]$ in (6.46). This proves the convergence in distribution of $\left(F_{n}\left(\frac{\left\lfloor s_{1} n\right\rfloor}{n}\right), \ldots, F_{n}\left(\frac{\left\lfloor s_{k} n\right\rfloor}{n}\right)\right.$. Finally, as $F_{n}(s)-F_{n}\left(\frac{\lfloor s n\rfloor}{n}\right)$ converges to zero as $n \rightarrow \infty$ (for any $s \in[0,1]$ and $\mathbb{P}$-almost-surely), we get the convergence of $\left(F_{n}\left(s_{1}\right), \ldots, F_{n}\left(s_{k}\right)\right)$.

Tightness. Proving tightness of the family $\left(F_{n}(\cdot)\right)_{n \geq 1}$ actually reduces to proving that for all $\varepsilon, \eta>0$, there exists $\delta>0$ such that for $n$ large enough,

$$
\mathbb{P}\left[\exists t \in[0,1-\delta]: \mathrm{P}\left(\sigma \wedge H_{\mathbb{Z}^{-}}>n(t+\delta) \mid \sigma \wedge H_{\mathbb{Z}^{-}}>n t\right) \leq \exp (-\eta N)\right] \leq \varepsilon .
$$


Let us first assume that

$$
\lim _{C \rightarrow \infty} \limsup _{n \rightarrow \infty} \mathbb{P}\left[B_{1}(C, n)^{c}\right]=0
$$

where

$$
B_{1}(C, n)=\left\{\forall t \in[0,1]: \mathrm{P}\left(H_{\tau_{C N}} \leq n t \mid \sigma \wedge H_{0}>n t\right) \leq \frac{1}{2}\right\},
$$

so that we can fix some $C>0$ such that $\mathbb{P}\left[B_{1}(C, n)\right] \geq 1-\varepsilon$ for $n$ large enough. For $\nu>0$, we define $a=\frac{\nu \eta}{\lambda}$ and consider the event

$$
B_{2}(\nu, n)=\left\{\forall x \leq C N, \exists y:|y-x| \leq a N, T_{y} \geq\left(a^{2} N\right)^{1 / \gamma}\right\} .
$$

Let us also assume for the moment that

$$
\lim _{\nu \rightarrow 0} \liminf _{n \rightarrow \infty} \mathbb{P}\left[B_{2}(\nu, n)\right]=1 .
$$

We can thus fix $\nu \in\left(0, \frac{1}{4}\right)$ such that $\mathbb{P}\left[B_{2}(\nu, n)^{c}\right]<\varepsilon$ for $n$ large enough. Finally, define

$$
B_{3}(n):=\left\{\forall 0 \leq i \leq C / a,-\frac{1}{N} \log \mathrm{P}_{\tau_{a i N}}\left(H_{\tau_{a(i+1) N}}<\sigma \wedge H_{\tau_{a i N}}\right)<2 a \lambda\right\}
$$

By Proposition $2.1 \mathbb{P}\left(B_{3}(n)\right)>1-\varepsilon$ for $n$ large enough. Therefore for $n$ large enough, the left-hand side of (B.1) is smaller than

(B.7)

$\mathbb{P}\left[\exists t \in[0,1-\delta]: \mathrm{P}\left(\sigma \wedge H_{\mathbb{Z}^{-}}>n(t+\delta) \mid \sigma \wedge H_{\mathbb{Z}^{-}}>n t\right) \leq e^{-\eta N}, B_{1}(C, n) \cap B_{2}(\nu, n) \cap B_{3}(n)\right]+3 \varepsilon$.

On $B_{1}(C, n) \cap B_{2}(\nu, n) \cap B_{3}(n)$, for all $t \in[0,1-\delta]$, we get by the Markov property applied at time $n t$ that

$$
\mathrm{P}\left(\sigma \wedge H_{\mathbb{Z}^{-}}>n(t+\delta) \mid \sigma \wedge H_{\mathbb{Z}^{-}}>n t\right) \geq \frac{1}{2} \inf _{x \in\left\{0, \ldots, \tau_{C N}\right\}} \mathrm{P}_{x}\left(\sigma \wedge H_{\mathbb{Z}^{-}}>\delta n\right) .
$$

By reproducing the strategy used in (4.8), we obtain for all $x \in\left\{0, \ldots, \tau_{C N}\right\}$

$$
\mathrm{P}_{x}\left(\sigma \wedge H_{\mathbb{Z}^{-}}>\delta n\right) \geq \exp \left[-N\left(2 \nu \eta+\frac{\delta \pi^{2}}{2(\nu \eta)^{4 / \gamma}}+o(1)\right)\right] .
$$

As $2 \nu<1$, we obtain by choosing $\delta>0$ small enough that

$$
\mathrm{P}\left(\sigma \wedge H_{\mathbb{Z}^{-}}>n(t+\delta) \mid \sigma \wedge H_{\mathbb{Z}^{-}}>n t\right)>e^{-\eta N} .
$$

It remains to prove (B.2) and (B.5).

Proof of (B.2). As

$$
P\left(H_{\tau_{C N}} \leq n t, \sigma \wedge H_{\mathbb{Z}^{-}}>n t\right) \leq \exp (-\beta C N)
$$

and

$$
P\left(\sigma \wedge H_{\mathbb{Z}^{-}}>n t\right) \geq P\left(\sigma \wedge H_{\mathbb{Z}^{-}}>n\right)
$$

for all $t \in[0,1]$,

$$
P\left(H_{\tau_{C N}} \leq n t \mid \sigma \wedge H_{\mathbb{Z}^{-}}>n t\right) \leq \exp \left[-N\left(\beta C-F_{n}(1)\right] .\right.
$$

As a consequence,

$$
\limsup _{n \rightarrow \infty} \mathbb{P}\left(\exists t \in[0,1]: P\left(H_{\tau_{C N}} \leq n t \mid \sigma \wedge H_{\mathbb{Z}^{-}}>n t\right)>\frac{1}{2}\right) \leq \mathbb{P}(F \geq \beta C),
$$

and this last quantity goes to 0 as $C \rightarrow+\infty$.

Proof of (B.5). For $n$ large enough,

$$
\mathbb{P}\left(\exists k \leq a N: T_{k} \geq\left(a^{2} N\right)^{1 / \gamma}\right) \geq 1-\exp \left(-\frac{c_{\tau}}{2 a}\right) .
$$


We cut the $C N$ first obstacles into $C / a$ disjoint intervals, each of them containing $a N$ obstacles, so that, by independence,

$$
\mathbb{P}\left[B_{2}(\nu, n)\right] \geq\left[1-\exp \left(-\frac{c_{\tau}}{2 a}\right)\right]^{C / a},
$$

and this last quantity goes to 1 if $a$ (or equivalently $\nu$ ) goes to 0 .

\section{REFERENCES}

[1] J. Alvarez and E. J. Janse van Rensburg. Directed paths in a layered environment. J. Phys. A, 41(46):465003, 40, 2008.

[2] S. Asmussen. Applied probability and queues, volume 51 of Applications of Mathematics (New York). Springer-Verlag, New York, second edition, 2003. Stochastic Modelling and Applied Probability.

[3] Serge Aubry and Gilles André. Analyticity breaking and Anderson localization in incommensurate lattices. In Group theoretical methods in physics (Proc. Eighth Internat. Colloq., Kiryat Anavim, 1979), volume 3 of Ann. Israel Phys. Soc., pages 133-164. Hilger, Bristol, 1980.

[4] F. Caravenna and N. Pétrélis. Depinning of a polymer in a multi-interface medium. Electron. J. Probab., 14:no. 70, 2038-2067, 2009.

[5] F. Caravenna and N. Pétrélis. A polymer in a multi-interface medium. Ann. Appl. Probab., 19(5):18031839,2009

[6] F. den Hollander. Random polymers, volume 1974 of Lecture Notes in Mathematics. Springer-Verlag, Berlin, 2009. Lectures from the 37th Probability Summer School held in Saint-Flour, 2007.

[7] J. Ding and C. Xu. Localization for random walks among random obstacles in a single Euclidean ball. ArXiv e-prints, July 2018.

[8] R. Durrett. Probability: theory and examples, volume 31 of Cambridge Series in Statistical and Probabilistic Mathematics. Cambridge University Press, Cambridge, fourth edition, 2010.

[9] William Feller. An introduction to probability theory and its applications. Vol. I. Third edition. John Wiley \& Sons, Inc., New York-London-Sydney, 1968.

[10] G. Giacomin. Random polymer models. Imperial College Press, London, 2007.

[11] G. Giacomin. Disorder and critical phenomena through basic probability models, volume 2025 of Lecture Notes in Mathematics. Springer, Heidelberg, 2011. Lecture notes from the 40th Probability Summer School held in Saint-Flour, 2010, École d'Été de Probabilités de Saint-Flour. [Saint-Flour Probability Summer School].

[12] R. Gouet, F. J. López, and G. Sanz. Central limit theorems for the number of records in discrete models. Adv. in Appl. Probab., 37(3):781-800, 2005.

[13] W. König. The parabolic Anderson model. Pathways in Mathematics. Birkhäuser/Springer, 2016. Random walk in random potential.

[14] V. V. Petrov. Limit theorems of probability theory, volume 4 of Oxford Studies in Probability. The Clarendon Press, Oxford University Press, New York, 1995. Sequences of independent random variables, Oxford Science Publications.

[15] A. Rényi. Théorie des éléments saillants d'une suite d'observations. Ann. Fac. Sci. Univ. ClermontFerrand No., 8:7-13, 1962.

[16] S. I. Resnick. Extreme values, regular variation and point processes. Springer Series in Operations Research and Financial Engineering. Springer, New York, 2008. Reprint of the 1987 original.

[17] E. Seneta. Non-negative matrices and Markov chains. Springer Series in Statistics. Springer, New York, 2006. Revised reprint of the second (1981) edition [Springer-Verlag, New York; MR0719544].

[18] A.-S. Sznitman. Brownian motion, obstacles and random media. Springer Monographs in Mathematics. Springer-Verlag, Berlin, 1998.

(J. Poisat) Université Paris-Dauphine, CNRS, UMR [7534], CEREMADE, PSL Research UniVERSITY, 75016 PARIS, FrANCE

E-mail address: poisat@ceremade.dauphine.fr

(F. Simenhaus) Université Paris-Dauphine, CNRS, UMR [7534], CEREMAde, 75016 Paris, FRANCE

E-mail address: simenhaus@ceremade.dauphine.fr 